\title{
Asymptotic Analysis of a Nonlinear Parabolic Problem Modelling Miscible Compressible Displacement in Porous Media
}

\author{
Catherine Choquet
}

\begin{abstract}
We study the asymptotic behavior, with respect to high Peclet numbers, of a model describing a compressible and miscible displacement in a porous medium. The transport of mass is then described by a nonlinear, fully coupled and degenerate parabolic system. Using non-classical estimates and renormalization tools, we prove existence of relevant weak solutions for the limit problem.
\end{abstract}

Mathematics Subject Classification (2000). 35K60, 35K65, 35B40, 76S05, $35 \mathrm{~K} 57$.

Keywords. Nonlinear degenerate parabolic system; asymptotic analysis; miscible compressible displacement; porous media.

\section{Introduction and main result}

We consider the displacement of two miscible species transported by a compressible flow in a porous medium. Our aim is the study of the main mechanisms governing for instance a nuclear waste-disposal problem, that is convection versus diffusion and dispersion effects at high Peclet numbers, for the critical settings of high Darcy rate of flow when the permeability of the rock is quite big. Let $\Omega$ be a domain of $\mathbb{R}^{N}, N=2$ or 3 , with smooth $\mathcal{C}^{1}$ boundary $\Gamma$. The unit normal pointing outward $\Omega$ is denoted by $\nu$. The time interval of interest is $(0, T), T>0$. We set $\Omega_{T}=\Omega \times(0, T)$ and $\Gamma_{T}=\Gamma \times(0, T)$. We denote by $p$ the pressure and by $c$ the mass concentration of one of the two components of the mixture. The Darcy velocity is designated by $\underline{u}$. Due to the mass and energy conservation (cf. $[7,14,16])$, 
the flow is governed by the following system satisfied in $\Omega_{T}$.

$$
\begin{aligned}
\phi(x) \partial_{t} p+\operatorname{div}(\underline{u}) & =0, \quad \underline{u}=-\frac{k(x)}{\mu(c)} \nabla p, \\
\phi(x) \partial_{t} c+\underline{u} \cdot \nabla c-\operatorname{div}(\mathcal{D}(\underline{u}) \nabla c) & =0 .
\end{aligned}
$$

Note that the second part of Eq. (1.1) is the classical Darcy law for porous media. We neglect the gravitational terms for sake of clarity in the estimates below. However no significant mathematical question arise when gravitational effects are included. This model takes into account the crucial influence of the concentration on the viscosity. A strong coupling is then induced in the system by the viscosity $\mu$ which is concentration dependent. The porosity and the permeability of the medium are respectively denoted $\phi$ and $k$. The diffusion effects are modelized by the tensor

$$
\mathcal{D}(\underline{u})=\phi\left(D_{m} I d+D_{p}(\underline{u})\right)=\phi\left(D_{m} I d+|\underline{u}|\left(\alpha_{L} \mathcal{E}(\underline{u})+\alpha_{T}(I d-\mathcal{E}(\underline{u}))\right)\right),
$$

where $\mathcal{E}(\underline{u})_{i j}=\underline{u}_{i} \underline{u}_{j} /|\underline{u}|^{2}, \alpha_{L}$ and $\alpha_{T}$ are the longitudinal and transverse dispersion constants and $D_{m}$ is the molecular diffusion.

At high Peclet numbers, the effects of molecular diffusion become negligible with respect to the dispersions ones (see for instance $[4,7,15]$ ). Therefore, we now set $D_{m}=\varepsilon$ and we aim to study the asymptotic behavior of the system (1.1)-(1.2) as $\varepsilon \rightarrow 0$ assuming that

$$
0<\alpha_{T} \leq \alpha_{L} .
$$

We thus consider the following equations in $\Omega_{T}$.

$$
\begin{aligned}
\phi \partial_{t} p_{\varepsilon}+\operatorname{div}\left(\underline{u}_{\varepsilon}\right) & =0, \quad \underline{u}_{\varepsilon}=-\frac{k}{\mu\left(c_{\varepsilon}\right)} \nabla p_{\varepsilon}, \\
\phi \partial_{t} c_{\varepsilon}+\underline{u}_{\varepsilon} \cdot \nabla c_{\varepsilon}-\operatorname{div}\left(\phi\left(\varepsilon I d+D_{p}\left(\underline{u}_{\varepsilon}\right)\right) \nabla c_{\varepsilon}\right) & =0 .
\end{aligned}
$$

On the one hand, Equation (1.4) is at the limit of degenerate parabolic type. It prevents from obtaining any estimate on the concentration gradient $\nabla c_{\varepsilon}$. On the other hand, the second main difficulty of the problem is its very strong coupling. In particular, the viscosity in Equation (1.3) is concentration dependent. The key idea of this work is thus an astute and new combination of Equations (1.3) and (1.4) to get an estimate for the space derivatives of the Darcy velocity $\underline{u}_{\varepsilon}$.

We assume that the porosity $\phi$ and the permeability $k$ are in $W^{1, \infty}(\Omega)$ and that they satisfy for some reals $0<\phi_{-} \leq \phi_{+}$and $0<k_{-} \leq k_{+}$

$$
\phi_{-} \leq \phi(x) \leq \phi_{+}, \quad k_{-} \leq k(x) \leq k_{+} \quad \text { a.e. in } \Omega .
$$

Since the diffusion has the following properties

$$
\begin{aligned}
\mathcal{D}\left(\underline{u}_{\varepsilon}\right) \xi \cdot \xi \geq \phi_{-}\left(\varepsilon+\alpha_{T}\left|\underline{u}_{\varepsilon}\right|\right)|\xi|^{2}, & \left|\mathcal{D}\left(\underline{u}_{\varepsilon}\right) \xi\right| \leq \phi_{+}\left(\varepsilon+\alpha_{L}\left|\underline{u}_{\varepsilon}\right|\right)|\xi|, \quad \forall \xi \in \mathbb{R}^{N},
\end{aligned}
$$


we emphasize that System (1.3)-(1.4) is of parabolic type while the limit system with $\varepsilon=0$ is of degenerate parabolic type. We assume that the viscosity $\mu$ is a convex function belonging to $W^{1, \infty}(0,1)$ and is bounded as follows.

$$
0<\mu_{-} \leq \mu(c) \leq \mu_{+}, \quad 0<\mu_{-} \leq \frac{\mu^{\prime \prime}(c)}{\mu^{2}(c)} \quad \forall c \in(0,1) .
$$

The inverse function $1 / \mu$ is also supposed convex. These assumptions are satisfied by the most usual models of viscosity. We cite in particular the Koval model [10] where $\mu$ is defined in $(0,1)$ by

$$
\mu(c)=\mu(0)\left(1+\left(M^{1 / 4}-1\right) c\right)^{-4},
$$

where $M=\mu(0) / \mu(1)$ is the mobility ratio. Our arguments are also valid for the Todd-Longstaff model [18], or the Fayers model [8] which are of the form $\mu(c)=\left(M^{\prime} c+M^{\prime \prime}(1-c)\right)^{-4}$, where $M^{\prime}$ and $M^{\prime \prime}$ are some nonnegative constants. A model of the form $\mu(c)=\mu_{0}+\mu_{1}\left(c-c_{r e f}\right)$ is sometimes used (cf. [16]), but it would bring less difficulty in this work. For sake of convenience, the function $\mu$ is continuously extended to $\mathbb{R}$. System (1.3)-(1.4) is completed by the following initial and boundary conditions.

$$
\begin{aligned}
& \underline{u}_{\varepsilon} \cdot \nu=0 \quad \text { on } \quad \Gamma_{T}, \quad p_{\varepsilon}(x, 0)=p_{\text {init }}(x) \text { in } \Omega, \\
& \mathcal{D}\left(\underline{u}_{\varepsilon}\right) \nabla c_{\varepsilon} \cdot \nu=0 \quad \text { on } \quad \Gamma_{T}, \quad c_{\varepsilon}(x, 0)=c_{\text {init }}(x) \text { in } \Omega \text {. }
\end{aligned}
$$

The initial data $p_{\text {init }} \in H^{1}(\Omega)$ and $c_{\text {init }} \in L^{\infty}(\Omega)$ satisfy

$$
\begin{aligned}
0 \leq c_{\text {init }}(x) & \leq 1 \quad \text { a.e. in } \quad \Omega, \\
\int_{\Omega} T_{2^{n}, 2^{n+1}}\left(\left|\nabla p_{\text {init }}\right|^{2}\right) d x & \leq C e^{-2^{n} T} \quad \forall n \in \mathbb{N},
\end{aligned}
$$

where the truncation function $T_{2^{n}, 2^{n+1}}$ is defined in $\mathbb{R}$ by

$$
T_{2^{n}, 2^{n+1}}(x)=\left\{\begin{array}{ll}
x & \text { if } 2^{2 n} \leq x \leq 2^{2 n+2} \\
0 & \text { elsewhere }
\end{array},\right.
$$

Assumption (1.11) is used to control the dispersive effects due to the Darcy velocity (see Section 3 below). Note that $(1.11)$ is obviously fulfilled if $p_{\text {init }} \in W^{1, \infty}(\Omega)$.

For any fixed $\varepsilon>0,[5]$ proves the following existence result.

Theorem 1. Assuming (1.5)-(1.11), Problem (1.3)-(1.4), (1.8)-(1.9) admits a weak solution $\left(p_{\varepsilon}, c_{\varepsilon}\right)$ satisfying

i) the function $p_{\varepsilon} \in L^{\infty}\left(0, T ; L^{2}(\Omega)\right) \cap L^{2}\left(0, T ; H^{1}(\Omega)\right)$ is solution of $(1.1)$, (1.8) verified in the space $L^{2}\left(0, T ; H^{-1}(\Omega)\right)$;

ii) the function $c_{\varepsilon} \in L^{\infty}\left(\Omega_{T}\right) \cap L^{2}\left(0, T ; H^{1}(\Omega)\right)$ is such that $0 \leq c_{\varepsilon}(x, t) \leq 1$ a.e. in $\Omega_{T}$.

The aim of the present paper is to state the following convergence and existence result. 
Theorem 2. Under the aforementioned hypotheses, there exists some functions $p \in E^{\infty}\left(0, T ; L^{2}(\Omega)\right) \cap L^{2}\left(0, T ; H^{1}(\Omega)\right), \underline{u} \in L^{2}\left(\Omega_{T}\right) \cap L^{\theta}\left(0, T ; W^{1, \theta}(\Omega)\right)$ with $\theta<$ $(6 N+12) /(5 N+8)$, and $c \in L^{\infty}\left(\Omega_{T}\right)$ such that, up to extracted subsequences, the following convergences hold true as $\varepsilon \rightarrow 0$.

$$
\begin{aligned}
p_{\varepsilon} & \rightarrow p \quad \text { a.e. in } \quad \Omega_{T}, \quad \partial_{t} p_{\varepsilon} \rightarrow \partial_{t} p \quad \text { weakly in } L^{\theta}\left(\Omega_{T}\right), \\
\nabla p_{\varepsilon} & \rightarrow \nabla p \quad \text { weakly in }\left(L^{2}\left(\Omega_{T}\right)\right)^{N}, \\
c_{\varepsilon} & \rightarrow c * \text {-weakly in } L^{\infty}\left(\Omega_{T}\right) .
\end{aligned}
$$

Moreover, $(p, c)$ is a weak solution of the following degenerate parabolic problem.

$$
\begin{aligned}
& \phi \partial_{t} p+\operatorname{div}(\underline{u})=0 \quad \text { in } \quad \Omega_{T}, \quad \text { where } \quad \underline{u}=-\frac{k}{\mu(c)} \nabla p, \\
& \phi \partial_{t} c+\underline{u} \cdot \nabla c-\operatorname{div}\left(\phi D_{p}(\underline{u}) \nabla c\right)=0 \quad \text { in } \Omega_{T}, \\
& \underline{u} \cdot \nu=0 \quad \text { on } \quad \Gamma_{T}, \quad p(x, 0)=p_{\text {init }}(x) \text { in } \Omega, \\
& D_{p}(\underline{u}) \nabla c \cdot \nu=0 \quad \text { on } \quad \Gamma_{T}, \quad c(x, 0)=c_{\text {init }}(x) \quad \text { in } \Omega .
\end{aligned}
$$

Let us mention some papers dealing with this problem. Amirat, Hamdache and Ziani consider in [1] the parabolic-hyperbolic problem with $D_{m}=D_{p}(\underline{u})=0$ when the viscosity $\mu$ is constant. The more realistic case $D_{m}=0, D_{p}(\underline{u}) \neq 0$ and $\mu=\mu(c)$ is treated by Amirat and Ziani in [3] for an incompressible flow, that is when the divergence of the Darcy velocity $\underline{u}$ is fixed and then completely controlled. The one-dimensional case has been more studied. We mention in particular Amirat and Moussaoui who obtain in [2] an existence result for a model without diffusion and dispersion but for a constant viscosity, and Choquet who considers in [6] the asymptotic behavior of the model with respect to law Peclet numbers when the viscosity is concentration dependent.

The paper is organized as follows. In Section 2, we begin by deriving classical energy estimates. To overcome the lake of regularity of the pressure $p_{\varepsilon}$, we then introduce a regularized pressure problem. In Section 3, combining astutely this regularized problem with the concentrations one, original test functions and renormalization arguments, we obtain an estimate in a space $L^{\theta}\left(0, T ; W^{1, \theta}(\Omega)\right)$ of the velocity $\underline{u}_{\varepsilon}$. Section 4 is finally devoted to the convergences results.

\section{First a priori estimates and introduction of an auxiliary regularized problem}

We begin by giving the results which can be obtained by classical energy estimates on Pb. (1.3), (1.8).

Lemma 1. We claim that the following uniform estimates hold true.

$$
\left\|p_{\varepsilon}\right\|_{L^{\infty}\left(0, T ; L^{2}(\Omega)\right)}+\left\|p_{\varepsilon}\right\|_{L^{2}\left(0, T ; H^{1}(\Omega)\right)} \leq C, \quad\left\|\underline{u}_{\varepsilon}\right\|_{\left(L^{2}\left(\Omega_{T}\right)\right)^{N}} \leq C .
$$

Furthermore, the function $\phi \partial_{t} p_{\varepsilon}$ is uniformly bounded in $L^{2}\left(0, T ; H^{-1}(\Omega)\right)$ and the sequence $\left(p_{\varepsilon}\right)$ is compact in $L^{2}\left(\Omega_{T}\right)$. 
Proof. We multiply Eq. (1.3) by $p_{\varepsilon}$ and we integrate over $\Omega$. Integrating by parts, we obtain

$$
\frac{1}{2} \frac{d}{d t} \int_{\Omega} \phi\left|p_{\varepsilon}(\cdot, t)\right|^{2} d x+\int_{\Omega} \frac{k}{\mu\left(c_{\varepsilon}\right)} \nabla p_{\varepsilon} \cdot \nabla p_{\varepsilon} d x=0 .
$$

Since $\phi(x) \geq \phi_{-}>0$ and $k / \mu\left(c_{\varepsilon}\right) \geq k_{-} / \mu_{+}$almost everywhere in $\Omega$, the latter relation and the Gronwall lemma give the first estimates of Lemma 1. Eq. (1.3) then directly implies that $\phi \partial_{t} p_{\varepsilon}$ is uniformly bounded in $L^{2}\left(0, T ; H^{-1}(\Omega)\right)$. Since the embedding $L^{2}(\Omega) \subset\left(H^{1}(\Omega)\right)^{\prime}$ is compact, we claim using a compactness result of Aubin's type (see [17]) that $\left(\phi p_{\varepsilon}\right)$ is sequentially compact in $L^{2}\left(0, T ;\left(H^{1}(\Omega)\right)^{\prime}\right)$. We thus can pass to the limit $\varepsilon \rightarrow 0$ in $\left\langle\phi p_{\varepsilon}, p_{\varepsilon}\right\rangle_{L^{2}\left(0, T ;\left(H^{1}(\Omega)\right)^{\prime}\right) \times L^{2}\left(0, T ; H^{1}(\Omega)\right)}$. Since $\phi(x) \geq \phi_{-}>0$ almost everywhere in $\Omega$, the compactness of the sequence $\left(p_{\varepsilon}\right)$ in $L^{2}\left(\Omega_{T}\right)$ follows.

We now perform energy estimates on the concentration problem (1.4), (1.9).

Lemma 2. The sequence $\left(c_{\varepsilon}\right)$ is uniformly bounded in the space $L^{\infty}\left(\Omega_{T}\right)$ and the gradients satisfy the following uniform estimate.

$$
\left\|\left(\varepsilon^{1 / 2}+\left|\underline{u}_{\varepsilon}\right|^{1 / 2}\right) \nabla c_{\varepsilon}\right\|_{\left(L^{2}\left(\Omega_{T}\right)\right)^{N}} \leq C .
$$

Proof. We begin by recalling that the concentration $c_{\varepsilon}$ is physically admissible in the sense that $0 \leq c_{\varepsilon}(x, t) \leq 1$ almost everywhere in $\Omega_{T}$. The sequence $\left(c_{\varepsilon}\right)$ is then uniformly bounded in $L^{\infty}\left(\Omega_{T}\right)$. We now multiply Eq. (1.4) by $c_{\varepsilon}$ and we integrate over $\Omega$. We obtain

$$
\frac{1}{2} \frac{d}{d t} \int_{\Omega} \phi\left|c_{\varepsilon}(\cdot, t)\right|^{2} d x+\int_{\Omega} \mathcal{D}_{\varepsilon}\left(\underline{u}_{\varepsilon}\right) \nabla c_{\varepsilon} \cdot \nabla c_{\varepsilon} d x+\int_{\Omega}\left(\underline{u}_{\varepsilon} \cdot \nabla c_{\varepsilon}\right) c_{\varepsilon} d x=0 .
$$

Since $c_{\varepsilon}$ is uniformly bounded in $L^{\infty}\left(\Omega_{T}\right)$, we can write using the Cauchy-Schwarz and Young inequalities and Lemma 1

$$
\begin{aligned}
\left|\int_{\Omega}\left(\underline{u}_{\varepsilon} \cdot \nabla c_{\varepsilon}\right) c_{\varepsilon} d x\right| & \leq C \int_{\Omega}\left|\underline{u}_{\varepsilon}\right| d x+\frac{\phi_{-} \alpha_{T}}{2} \int_{\Omega}\left|\underline{u}_{\varepsilon}\right|\left|\nabla c_{\varepsilon}\right|^{2} d x \\
& \leq C(t)+\frac{\phi_{-} \alpha_{T}}{2} \int_{\Omega}\left|\underline{u}_{\varepsilon}\right|\left|\nabla c_{\varepsilon}\right|^{2} d x
\end{aligned}
$$

where the quantity $C(t)$ is uniformly bounded in $L^{1}(0, T)$. Using Assumption (1.6), Relation (2.1) thus leads to

$$
\frac{1}{2} \frac{d}{d t} \int_{\Omega} \phi\left|c_{\varepsilon}(\cdot, t)\right|^{2} d x+\int_{\Omega} \phi_{-}\left(\frac{\alpha_{T}}{2}\left|\underline{u}_{\varepsilon}\right|+\varepsilon\right)\left|\nabla c_{\varepsilon}\right|^{2} d x \leq C(t) .
$$

Bearing in mind the property $\phi(x) \geq \phi_{-}>0$ a.e. in $\Omega$, we prove Lemma 2 using the Gronwall lemma.

At this step, we clearly do not have enough compactness results to pass to the limit in $\mathrm{Pb}$. (1.3)-(1.4), (1.8)-(1.9). One of the main difficulties is the lake of regularity of the pressure $p_{\varepsilon}$ which does not allow other estimates than the classical ones developped in Lemma 1. One of the main tool of our work is thus the definition of an auxiliary "regularized" pressure problem. To this aim, we 
extend the concentration $c_{\varepsilon}$ in a function $\widetilde{c_{\varepsilon}} \in L^{2}\left(\mathbb{R} ; H^{1}(\mathbb{R})\right)$. We denote by $\rho$ a nonnegative function of $\mathcal{C}^{\infty}\left(\mathbb{R}^{N+1}\right)$, with support in the unit ball and such that $\int_{\mathbb{R}^{N+1}} \rho(x, t) d x d t=1$. For any $\alpha>0$, we then set $\rho^{\alpha}(x, t)=\rho(x / \alpha, t / \alpha) / \alpha^{N+1}$. We define a regularized concentration $c_{\varepsilon}^{\alpha}$ using a convolution product

$$
\widetilde{c_{\varepsilon}^{\alpha}}(x, t)=\left(\rho^{\alpha} * \widetilde{c_{\varepsilon}}\right)(x, t)=\int_{\mathbb{R}^{N+1}} \rho^{\alpha}(y, u) \widetilde{c_{\varepsilon}}(x-y, t-u) d y d u .
$$

We denote by $c_{\varepsilon}^{\alpha}$ the restriction of $\widetilde{c_{\varepsilon}^{\alpha}}$ to $\Omega_{T}$. The function $c_{\varepsilon}^{\alpha} \in \mathcal{C}^{\infty}\left(\bar{\Omega}_{T}\right)$ satisfies as $\alpha \rightarrow 0$

$$
c_{\varepsilon}^{\alpha} \rightarrow c_{\varepsilon} \quad \text { strongly in } L^{2}\left(0, T ; H^{1}(\Omega)\right) .
$$

We also note that $\left(c_{\varepsilon}^{\alpha}\right)$ is uniformly bounded in $L^{\infty}\left(\Omega_{T}\right)$. Now, for any $\alpha>0$, we associate with $c_{\varepsilon}^{\alpha}$ a function $p_{\varepsilon}^{\alpha}$ solution of the following problem.

$$
\begin{aligned}
\phi \partial_{t} p_{\varepsilon}^{\alpha}+\operatorname{div}\left(\underline{u}_{\varepsilon}^{\alpha}\right) & =0, \quad \underline{u}_{\varepsilon}^{\alpha}=-\frac{k}{\mu\left(c_{\varepsilon}^{\alpha}\right)} \nabla p_{\varepsilon}^{\alpha} \quad \text { in } \Omega_{T}, \\
\underline{u}_{\varepsilon}^{\alpha} \cdot \nu=0 \quad \text { on } \quad \Gamma_{T}, \quad p_{\varepsilon}^{\alpha}(x, 0) & =p_{\text {init }}^{\alpha}(x) \quad \text { in } \Omega,
\end{aligned}
$$

where the initial pressure $p_{\text {init }}^{\alpha}$ is a function of $\mathcal{C}^{2}(\bar{\Omega})$ such that

$$
p_{\text {init }}^{\alpha} \rightarrow p_{\text {init }} \quad \text { strongly in } \quad H^{1}(\Omega) .
$$

The existence of a unique solution $p_{\varepsilon}^{\alpha}$ to the parabolic problem with smooth coefficients (2.3)-(2.4) is classical (see for instance [11]). Moreover, we assert that the following results hold true.

\section{Lemma 3.}

(i) For any $\alpha>0$, the regularized pressure $p_{\varepsilon}^{\alpha}$ belongs to the space $L^{\infty}(0, T$; $\left.H^{1}(\Omega)\right) \cap H^{1}\left(0, T ; L^{2}(\Omega)\right) \cap L^{2}\left(0, T ; W^{2,2}(\Omega)\right)$.

(ii) The following estimates are uniform with respect to $\alpha$ and $\varepsilon$ :

$$
\begin{aligned}
\left\|p_{\varepsilon}^{\alpha}\right\|_{L^{2}\left(0, T ; H^{1}(\Omega)\right)}+\left\|p_{\varepsilon}^{\alpha}\right\|_{L^{\infty}\left(0, T ; L^{2}(\Omega)\right)} & \leq C, \quad\left\|\underline{u}_{\varepsilon}^{\alpha}\right\|_{\left(L^{2}(\Omega)\right)^{N}} \leq C, \\
\left\|\phi \partial_{t} p_{\varepsilon}^{\alpha}\right\|_{L^{2}\left(0, T ; H^{-1}(\Omega)\right)} & \leq C .
\end{aligned}
$$

(iii) As $\alpha \rightarrow 0$, the sequence $\left(p_{\varepsilon}^{\alpha}\right)$ converges strongly in $L^{2}\left(\Omega_{T}\right)$ to the solution $p_{\varepsilon}$ of Problem (1.3), (1.8). Furthermore

$$
\nabla p_{\varepsilon}^{\alpha} \rightarrow \nabla p_{\varepsilon} \quad \text { strongly in } \quad\left(L^{2}\left(\Omega_{T}\right)\right)^{N} \quad \text { and a.e. in } \quad \Omega_{T} .
$$


Proof. We begin by multiplying Eq. (2.3) by $\partial_{t} p_{\varepsilon}^{\alpha}$. Integrating by parts over $\Omega$, we obtain

$$
\begin{aligned}
\int_{\Omega} \phi\left|\partial_{t} p_{\varepsilon}^{\alpha}\right|^{2} d x+\int_{\Omega} \frac{k}{\mu\left(c_{\varepsilon}^{\alpha}\right)} \nabla & p_{\varepsilon}^{\alpha} \cdot \partial_{t}\left(\nabla p_{\varepsilon}^{\alpha}\right) d x=\int_{\Omega} \phi\left|\partial_{t} p_{\varepsilon}^{\alpha}\right|^{2} d x \\
& +\frac{1}{2} \frac{d}{d t} \int_{\Omega} \frac{k}{\mu\left(c_{\varepsilon}^{\alpha}\right)} \nabla p_{\varepsilon}^{\alpha}(\cdot, t) \cdot \nabla p_{\varepsilon}^{\alpha}(\cdot, t) d x \\
& +\frac{1}{2} \int_{\Omega} \frac{\mu^{\prime}\left(c_{\varepsilon}^{\alpha}\right)}{\mu^{2}\left(c_{\varepsilon}^{\alpha}\right)} \partial_{t} c_{\varepsilon}^{\alpha} k \nabla p_{\varepsilon}^{\alpha} \cdot \nabla p_{\varepsilon}^{\alpha} d x \\
= & 0 .
\end{aligned}
$$

Since $\partial_{t} c_{\varepsilon}^{\alpha} \in \mathcal{C}^{\infty}\left(\bar{\Omega}_{T}\right)$, using the Gronwall lemma and the bounds of the porosity, permeability and viscosity given in (1.5) and (1.7), we then assert the existence of a quantity $C(\alpha)$ such that

$$
\left\|\partial_{t} p_{\varepsilon}^{\alpha}\right\|_{L^{2}\left(\Omega_{T}\right)}+\left\|p_{\varepsilon}^{\alpha}\right\|_{L^{\infty}\left(0, T ; H^{1}(\Omega)\right)} \leq C(\alpha) .
$$

It implies in particular that $\operatorname{div} \underline{u}_{\varepsilon}^{\alpha}=-\partial_{t} p_{\varepsilon}^{\alpha}$ is also bounded in $L^{2}\left(\Omega_{T}\right)$. Furthermore, since $\mu$ is a function of $W^{1, \infty}(0,1)$ continuously extended to $\mathbb{R}$ and since $k \in W^{1, \infty}(\Omega)$, curl $u_{\varepsilon}^{\alpha}$ is bounded like $\nabla c_{\varepsilon}^{\alpha} \wedge \nabla p_{\varepsilon}^{\alpha}$, that is in $\left(L^{2}\left(\Omega_{T}\right)\right)^{N}$. We conclude that $\underline{u}_{\varepsilon}^{\alpha}$ is bounded in $L^{2}\left(0, T ; H^{1}(\Omega)\right)$ and that there exists some constant $C(\alpha)$ such that

$$
\left\|p_{\varepsilon}^{\alpha}\right\|_{L^{2}\left(0, T ; W^{2,2}(\Omega)\right)} \leq C(\alpha) .
$$

The proof of the uniform estimates in item (ii) can be carried out exactly like in Lemma 1. Using an argument of Aubin's type, we conclude that $\left(\phi p_{\varepsilon}^{\alpha}\right)$ is sequentially compact in $L^{2}\left(0, T ;\left(H^{1}(\Omega)\right)^{\prime}\right)$. On the other hand the function $p_{\varepsilon}^{\alpha}$ is uniformly bounded in $L^{2}\left(0, T ; H^{1}(\Omega)\right)$. We thus can pass to the limit $\alpha \rightarrow$ 0 in the product $\left\langle\phi p_{\varepsilon}^{\alpha}, p_{\varepsilon}^{\alpha}\right\rangle_{L^{2}\left(0, T ;\left(H^{1}(\Omega)\right)^{\prime}\right) \times L^{2}\left(0, T ; H^{1}(\Omega)\right)}$. Since $\phi \geq \phi_{-}>0$ a.e. in $\Omega$, it proves the sequential compactness of $\left(p_{\varepsilon}^{\alpha}\right)$ in $L^{2}\left(\Omega_{T}\right)$. Using the other uniform estimates of (ii), we assert the existence of subsequences, not relabeled for convenience, and of functions $P_{\varepsilon} \in L^{\infty}\left(0, T ; L^{2}(\Omega)\right) \cap L^{2}\left(0, T ; H^{1}(\Omega)\right)$ and $\underline{U}_{\varepsilon} \in\left(L^{2}\left(\Omega_{T}\right)\right)^{N}$, such that when $\alpha \rightarrow 0$

$$
\begin{aligned}
& p_{\varepsilon}^{\alpha} \rightarrow P_{\varepsilon} \quad \text { weakly in } L^{2}\left(0, T ; H^{1}(\Omega)\right) \text { and a.e. in } \Omega_{T}, \\
& \underline{u}_{\varepsilon}^{\alpha} \rightarrow \underline{U}_{\varepsilon} \quad \text { weakly in }\left(L^{2}\left(\Omega_{T}\right)\right)^{N} .
\end{aligned}
$$

Since there is a subsequence of $\left(c_{\varepsilon}^{\alpha}\right)$ which converges almost everywhere in $\Omega_{T}$ to $c_{\varepsilon}$, we claim that the function $P_{\varepsilon}$ is a solution of

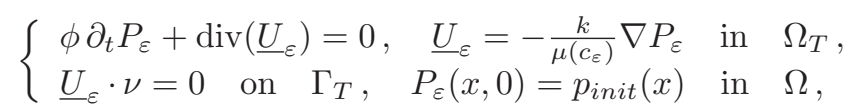

that is Problem (1.3), (1.8). Due to the uniqueness of the solution of this problem, we can ensure that the whole sequence $\left(p_{\varepsilon}^{\alpha}\right)$ converges to $P_{\varepsilon}=p_{\varepsilon}$ and that $\underline{U}_{\varepsilon}=\underline{u}_{\varepsilon}$. Now, let $t \in(0, T)$ and $\Omega_{t}=\Omega \times(0, t)$. Multiplying (2.3) by $p_{\varepsilon}^{\alpha}$, (1.3) by $p_{\varepsilon}$ and 
integrating by parts over $\Omega_{t}$, we obtain almost everywhere in $(0, T)$

$$
\begin{aligned}
& \frac{1}{2} \int_{\Omega} \phi\left|p_{\varepsilon}^{\alpha}(\cdot, t)\right|^{2} d x-\frac{1}{2} \int_{\Omega} \phi\left|p_{\text {init }}^{\alpha}(x)\right|^{2} d x+\int_{\Omega_{t}} \frac{k}{\mu\left(c_{\varepsilon}^{\alpha}\right)} \nabla p_{\varepsilon}^{\alpha} \cdot \nabla p_{\varepsilon}^{\alpha} d x d s=0, \\
& \frac{1}{2} \int_{\Omega} \phi\left|p_{\varepsilon}(\cdot, t)\right|^{2} d x-\frac{1}{2} \int_{\Omega} \phi\left|p_{\text {init }}(x)\right|^{2} d x+\int_{\Omega_{t}} \frac{k}{\mu\left(c_{\varepsilon}\right)} \nabla p_{\varepsilon} \cdot \nabla p_{\varepsilon} d x d s=0 .
\end{aligned}
$$

We subtract these relations and let $\alpha \rightarrow 0$. Since $p_{\varepsilon}^{\alpha}$ converges to $p_{\varepsilon}$ almost everywhere in $\Omega_{T}$, we get

$$
\lim _{\alpha \rightarrow 0} \int_{\Omega_{t}} \frac{k}{\mu\left(c_{\varepsilon}^{\alpha}\right)} \nabla p_{\varepsilon}^{\alpha} \cdot \nabla p_{\varepsilon}^{\alpha} d x d s=\int_{\Omega_{t}} \frac{k}{\mu\left(c_{\varepsilon}\right)} \nabla p_{\varepsilon} \cdot \nabla p_{\varepsilon} d x d s .
$$

Then, using the a.e. convergence of $\mu\left(c_{\varepsilon}^{\alpha}\right)$ to $\mu\left(c_{\varepsilon}\right)$ in $\Omega_{T}$, we compute

$$
\begin{aligned}
& \lim _{\alpha \rightarrow 0} \int_{\Omega_{t}} k\left(\frac{1}{\mu\left(c_{\varepsilon}^{\alpha}\right)^{1 / 2}} \nabla p_{\varepsilon}^{\alpha}-\frac{1}{\mu\left(c_{\varepsilon}\right)^{1 / 2}} \nabla p_{\varepsilon}\right) \\
& \cdot\left(\frac{1}{\mu\left(c_{\varepsilon}^{\alpha}\right)^{1 / 2}} \nabla p_{\varepsilon}^{\alpha}-\frac{1}{\mu\left(c_{\varepsilon}\right)^{1 / 2}} \nabla p_{\varepsilon}\right) d x d s=0 .
\end{aligned}
$$

Therefore, due to the properties of $k$ and $1 / \mu$, we conclude that

$$
\nabla p_{\varepsilon}^{\alpha} \rightarrow \nabla p_{\varepsilon}, \quad \underline{u}_{\varepsilon}^{\alpha} \rightarrow \underline{u}_{\varepsilon} \quad \text { a.e. in } \quad \Omega_{T} .
$$

This ends the proof of the lemma.

\section{Estimate of the Darcy velocity}

In this section, we use the auxiliary regularized problem (2.3)-(2.4) to obtain a stronger estimate on the Darcy velocity $\underline{u}_{\varepsilon}$. We aim to develop renormalisations arguments (see [13]) to estimate $\underline{u}_{\varepsilon}$ in $L^{\theta}\left(0, T ; W^{1, \theta}(\Omega)\right)$. One of the crucial ideas of the present paper is the astuteness in the proof of the following lemma.

Lemma 4. The following uniform estimates hold true for any $1 \leq i, j \leq N$

$$
\left\{\begin{array}{l}
\left\|\partial_{i} \underline{u}_{\varepsilon, j}\right\|_{L^{2}\left(B_{n}\right)}^{2} \leq 2^{n} C \text { for any } n \in \mathbb{N}, \\
\left\|\partial_{i} \underline{u}_{\varepsilon, j}\right\|_{L^{2}(B)}^{2} \leq C,
\end{array}\right.
$$

where the sets $B_{n}$ and $B$ are defined by $B_{n}=\left\{(x, t) \in \Omega_{T} ; 2^{n} \leq k^{\frac{1}{2}}(x)\left|\nabla p_{\varepsilon}(x, t)\right| \leq\right.$ $\left.2^{n+1}\right\}$ and $B=\left\{(x, t) \in \Omega_{T} ; 0 \leq k^{\frac{1}{2}}(x)\left|\nabla p_{\varepsilon}(x, t)\right| \leq 1\right\}$, so that $\Omega_{T}=B \cup$ $\left(\cup_{n \geq 0} B_{n}\right)$.

Proof. For any given reals $0<m<M$ and $\eta>0$ such that $m-\eta>0$, we consider a truncation function $T_{m, M}^{\eta} \in \mathcal{C}^{2}(\mathbb{R})$ such that

$$
T_{m, M}^{\eta}(x)=\left\{\begin{array}{lll}
0 & \text { if } & x \leq(m-\eta)^{2} \\
x & \text { if } & m^{2} \leq x \leq M^{2} \\
0 & \text { if } & x \geq(M+\eta)^{2}
\end{array} .\right.
$$

For sake of clarity, we omit the subscript $m, M$ and we set $T^{\eta}=T_{m, M}^{\eta}$. The idea of the proof is to combine astutely the concentration equation with the regularized 
pressure one. We begin by checking the existence of three integrals. On the one hand, we consider the term

$$
I_{1}=\int_{\Omega} \frac{\mu^{\prime}\left(c_{\varepsilon}\right)}{\mu^{2}\left(c_{\varepsilon}\right)} T_{\eta}\left(\left|k^{\frac{1}{2}} \nabla p_{\varepsilon}^{\alpha}\right|^{2}\right) \underline{u}_{\varepsilon} \cdot \nabla c_{\varepsilon} d x .
$$

Since $\underline{u}_{\varepsilon} \in\left(L^{2}\left(\Omega_{T}\right)\right)^{N}, \nabla c_{\varepsilon} \in\left(L^{2}\left(\Omega_{T}\right)\right)^{N}$ and the other integrated terms belong to $L^{\infty}\left(\Omega_{T}\right)$, we can ensure that the integral form $I_{1}$ belongs to $L^{1}(0, T)$. On the other hand, we define $I_{2}$ by

$$
I_{2}=\int_{\Omega} \frac{\mu^{\prime}\left(c_{\varepsilon}\right)}{\mu^{2}\left(c_{\varepsilon}\right)} \phi\left(\varepsilon+D_{p}\left(\underline{u}_{\varepsilon}\right)\right) \nabla c_{\varepsilon} \cdot T_{\eta}^{\prime}\left(\left|k^{\frac{1}{2}} \nabla p_{\varepsilon}^{\alpha}\right|^{2}\right) \nabla\left(\left|k^{\frac{1}{2}} \nabla p_{\varepsilon}^{\alpha}\right|^{2}\right) d x .
$$

We write $I_{2}$ in the following way

$$
\begin{aligned}
I_{2}= & \int_{\Omega} \frac{2 \mu^{\prime}\left(c_{\varepsilon}\right)}{\mu^{2}\left(c_{\varepsilon}\right)} \phi \varepsilon \nabla c_{\varepsilon} \cdot T_{\eta}^{\prime}\left(\left|k^{\frac{1}{2}} \nabla p_{\varepsilon}^{\alpha}\right|^{2}\right)\left(k^{\frac{1}{2}} \nabla p_{\varepsilon}^{\alpha} \cdot \nabla\right) k^{\frac{1}{2}} \nabla p_{\varepsilon}^{\alpha} \\
& +\int_{\Omega} \frac{2 \mu^{\prime}\left(c_{\varepsilon}\right)}{\mu^{2}\left(c_{\varepsilon}\right)} \phi D_{p}\left(\underline{u}_{\varepsilon}^{\alpha}\right) \nabla c_{\varepsilon} \cdot T_{\eta}^{\prime}\left(\left|k^{\frac{1}{2}} \nabla p_{\varepsilon}^{\alpha}\right|^{2}\right)\left(k^{\frac{1}{2}} \nabla p_{\varepsilon}^{\alpha} \cdot \nabla\right) k^{\frac{1}{2}} \nabla p_{\varepsilon}^{\alpha} \\
& +\int_{\Omega} \frac{2 \mu^{\prime}\left(c_{\varepsilon}\right)}{\mu^{2}\left(c_{\varepsilon}\right)} \phi\left(D_{p}\left(\underline{u}_{\varepsilon}\right)-D_{p}\left(\underline{u}_{\varepsilon}^{\alpha}\right)\right) \nabla c_{\varepsilon} \\
& \cdot T_{\eta}^{\prime}\left(\left|k^{\frac{1}{2}} \nabla p_{\varepsilon}^{\alpha}\right|^{2}\right)\left(k^{\frac{1}{2}} \nabla p_{\varepsilon}^{\alpha} \cdot \nabla\right) k^{\frac{1}{2}} \nabla p_{\varepsilon}^{\alpha} .
\end{aligned}
$$

We bear in mind that the term $T_{\eta}^{\prime}\left(\left|k^{\frac{1}{2}} \nabla p_{\varepsilon}^{\alpha}\right|^{2}\right)$ ensures that we work in a subdomain where the components of $\nabla p_{\varepsilon}^{\alpha}$, and then of $\underline{u}_{\varepsilon}^{\alpha}$ and $D_{p}\left(\underline{u}_{\varepsilon}^{\alpha}\right)$, belong to $L^{\infty}$. Then, since $\nabla c_{\varepsilon} \in\left(L^{2}\left(\Omega_{T}\right)\right)^{N}, p_{\varepsilon}^{\alpha} \in L^{2}\left(0, T ; W^{2,2}(\Omega)\right)$ and $k \in W^{1, \infty}(\Omega)$, the first two terms of the latter decomposition belong to $L^{1}(0, T)$. Let us now study the third one. We recall that by Lemma $3, \underline{u}_{\varepsilon}^{\alpha}$ converges to $\underline{u}_{\varepsilon}$ almost everywhere in $\Omega_{T}$. Thus, for a "sufficiently small" real $\alpha$, the set where $\left|D_{p}\left(\underline{u}_{\varepsilon}\right)-D_{p}\left(\underline{u}_{\varepsilon}^{\alpha}\right)\right|>1$ has a negligible measure. We thus consider that the term $D_{p}\left(\underline{u}_{\varepsilon}\right)-D_{p}\left(\underline{u}_{\varepsilon}^{\alpha}\right)$ in the third integral of (3.1) belongs to $\left(L^{\infty}\left(\Omega_{T}\right)\right)^{N^{2}}$. And the same tools as previously let us claim that this third integral and then $I_{2}$ are in the space $L^{1}(0, T)$. Finally, we consider $I_{3}$ defined by

$$
I_{3}=\int_{\Omega}\left(\frac{\mu^{\prime}\left(c_{\varepsilon}\right)}{\mu^{2}\left(c_{\varepsilon}\right)}\right)^{\prime} T_{\eta}\left(\left|k^{\frac{1}{2}} \nabla p_{\varepsilon}^{\alpha}\right|^{2}\right) \phi\left(\varepsilon+D_{p}\left(\underline{u}_{\varepsilon}\right)\right) \nabla c_{\varepsilon} \cdot \nabla c_{\varepsilon} d x .
$$

Using the same decomposition on the term $D_{p}\left(\underline{u}_{\varepsilon}\right)$ than in (3.1), we can check that $I_{3}$ also belongs to $L^{1}(0, T)$. We now note that an integration by parts gives

$$
I_{1}+I_{2}+I_{3}=I_{1}-\int_{\Omega} \operatorname{div}\left(\phi\left(\varepsilon+D_{p}\left(\underline{u}_{\varepsilon}\right)\right) \nabla c_{\varepsilon}\right) \frac{\mu^{\prime}\left(c_{\varepsilon}\right)}{\mu^{2}\left(c_{\varepsilon}\right)} T_{\eta}\left(\left|k^{\frac{1}{2}} \nabla p_{\varepsilon}^{\alpha}\right|^{2}\right) d x .
$$

Using the concentration equation (1.4) and the latter existence results for $I_{1}, I_{2}$ and $I_{3}$, we thus conclude that

$$
\int_{\Omega} \phi \partial_{t} c_{\varepsilon} \frac{\mu^{\prime}\left(c_{\varepsilon}\right)}{\mu^{2}\left(c_{\varepsilon}\right)} T_{\eta}\left(\left|k^{\frac{1}{2}} \nabla p_{\varepsilon}^{\alpha}\right|^{2}\right) d x=-I_{1}-I_{2}-I_{3} \in L^{1}(0, T) .
$$


We now note that

$$
\begin{aligned}
\int_{\Omega} \phi \partial_{t} c_{\varepsilon} \frac{\mu^{\prime}\left(c_{\varepsilon}\right)}{\mu^{2}\left(c_{\varepsilon}\right)} T_{\eta}\left(\left|k^{\frac{1}{2}} \nabla p_{\varepsilon}^{\alpha}\right|^{2}\right) d x= & -\frac{d}{d t} \int_{\Omega} \frac{\phi}{\mu\left(c_{\varepsilon}\right)} T_{\eta}\left(\left|k^{\frac{1}{2}} \nabla p_{\varepsilon}^{\alpha}\right|^{2}\right) d x \\
& +\int_{\Omega} \frac{\phi}{\mu\left(c_{\varepsilon}\right)} \partial_{t}\left(T_{\eta}\left(\left|k^{\frac{1}{2}} \nabla p_{\varepsilon}^{\alpha}\right|^{2}\right)\right) d x
\end{aligned}
$$

where

$$
\begin{aligned}
\frac{1}{2} \int_{\Omega} \frac{\phi}{\mu\left(c_{\varepsilon}\right)} \partial_{t}( & \left.T_{\eta}\left(\left|k^{\frac{1}{2}} \nabla p_{\varepsilon}^{\alpha}\right|^{2}\right)\right)=\int_{\Omega} \frac{\phi k}{\mu\left(c_{\varepsilon}\right)} T_{\eta}^{\prime}\left(\left|k^{\frac{1}{2}} \nabla p_{\varepsilon}^{\alpha}\right|^{2}\right) \nabla p_{\varepsilon}^{\alpha} \cdot \nabla\left(\partial_{t} p_{\varepsilon}^{\alpha}\right) \\
= & -\int_{\Omega} \operatorname{div}\left(\frac{\phi k}{\mu\left(c_{\varepsilon}\right)} T_{\eta}^{\prime}\left(\left|k^{\frac{1}{2}} \nabla p_{\varepsilon}^{\alpha}\right|^{2}\right) \nabla p_{\varepsilon}^{\alpha}\right) \partial_{t} p_{\varepsilon}^{\alpha} \\
= & -\int_{\Omega} \phi T_{\eta}^{\prime}\left(\left|k^{\frac{1}{2}} \nabla p_{\varepsilon}^{\alpha}\right|^{2}\right) \operatorname{div}\left(\frac{k}{\mu\left(c_{\varepsilon}\right)} \nabla p_{\varepsilon}^{\alpha}\right) \partial_{t} p_{\varepsilon}^{\alpha} \\
& -\int_{\Omega} \phi T_{\eta}^{\prime \prime}\left(\left|k^{\frac{1}{2}} \nabla p_{\varepsilon}^{\alpha}\right|^{2}\right)\left(\frac{k}{\mu\left(c_{\varepsilon}\right)} \nabla p_{\varepsilon}^{\alpha} \cdot \nabla\left(\left|\nabla p_{\varepsilon}^{\alpha}\right|^{2}\right)\right) \partial_{t} p_{\varepsilon}^{\alpha} \\
& -\int_{\Omega} T_{\eta}^{\prime}\left(\left|k^{\frac{1}{2}} \nabla p_{\varepsilon}^{\alpha}\right|^{2}\right)\left(\frac{k}{\mu\left(c_{\varepsilon}\right)} \nabla p_{\varepsilon}^{\alpha} \cdot \nabla \phi\right) \partial_{t} p_{\varepsilon}^{\alpha} \\
= & -\int_{\Omega} \phi T_{\eta}^{\prime}\left(\left|k^{\frac{1}{2}} \nabla p_{\varepsilon}^{\alpha}\right|^{2}\right)\left|\partial_{t} p_{\varepsilon}^{\alpha}\right|^{2} \\
& +\int_{\Omega} \phi T_{\eta}^{\prime}\left(\left|k^{\frac{1}{2}} \nabla p_{\varepsilon}^{\alpha}\right|^{2}\right) \operatorname{div}\left(\left(\frac{k}{\mu\left(c_{\varepsilon}^{\alpha}\right)}-\frac{k}{\mu\left(c_{\varepsilon}\right)}\right) \nabla p_{\varepsilon}^{\alpha}\right) \partial_{t} p_{\varepsilon}^{\alpha} \\
& -\int_{\Omega} \phi T_{\eta}^{\prime \prime}\left(\left|k^{\frac{1}{2}} \nabla p_{\varepsilon}^{\alpha}\right|^{2}\right) \times\left(\frac{k}{\mu\left(c_{\varepsilon}\right)} \nabla p_{\varepsilon}^{\alpha} \cdot \nabla\left(\left|\nabla p_{\varepsilon}^{\alpha}\right|^{2}\right)\right) \partial_{t} p_{\varepsilon}^{\alpha} \\
& -\int_{\Omega} T_{\eta}^{\prime}\left(\left|k^{\frac{1}{2}} \nabla p_{\varepsilon}^{\alpha}\right|^{2}\right)\left(\frac{k}{\mu\left(c_{\varepsilon}\right)} \nabla p_{\varepsilon}^{\alpha} \cdot \nabla \phi\right) \partial_{t} p_{\varepsilon}^{\alpha} .
\end{aligned}
$$

Therefore, using (3.2)-(3.3) and the latter relation, we conclude that

$$
\begin{aligned}
\frac{d}{d t} & \int_{\Omega} \frac{\phi}{\mu\left(c_{\varepsilon}\right)} T_{\eta}\left(\left|k^{\frac{1}{2}} \nabla p_{\varepsilon}^{\alpha}\right|^{2}\right) d x+2 \int_{\Omega} \phi T_{\eta}^{\prime}\left(\left|k^{\frac{1}{2}} \nabla p_{\varepsilon}^{\alpha}\right|^{2}\right)\left|\partial_{t} p_{\varepsilon}^{\alpha}\right|^{2} d x \\
& +\int_{\Omega}\left(\frac{\mu^{\prime \prime} \mu-2\left(\mu^{\prime}\right)^{2}}{\mu^{3}}\right)\left(c_{\varepsilon}\right) T_{\eta}\left(\left|k^{\frac{1}{2}} \nabla p_{\varepsilon}^{\alpha}\right|^{2}\right) \phi\left(\varepsilon+D_{p}\left(\underline{u}_{\varepsilon}\right)\right) \nabla c_{\varepsilon} \cdot \nabla c_{\varepsilon} d x \\
= & -\int_{\Omega} \frac{\mu^{\prime}\left(c_{\varepsilon}\right)}{\mu^{2}\left(c_{\varepsilon}\right)} T_{\eta}\left(\left|k^{\frac{1}{2}} \nabla p_{\varepsilon}^{\alpha}\right|^{2}\right) \underline{u}_{\varepsilon} \cdot \nabla c_{\varepsilon} d x \\
& -\int_{\Omega} \frac{\mu^{\prime}\left(c_{\varepsilon}\right)}{\mu^{2}\left(c_{\varepsilon}\right)} \phi\left(\varepsilon+D_{p}\left(\underline{u}_{\varepsilon}\right)\right) \nabla c_{\varepsilon} \cdot T_{\eta}^{\prime}\left(\left|k^{\frac{1}{2}} \nabla p_{\varepsilon}^{\alpha}\right|^{2}\right) \nabla\left(\left|k^{\frac{1}{2}} \nabla p_{\varepsilon}^{\alpha}\right|^{2}\right) d x \\
& +2 \int_{\Omega} \phi T_{\eta}^{\prime}\left(\left|k^{\frac{1}{2}} \nabla p_{\varepsilon}^{\alpha}\right|^{2}\right) \operatorname{div}\left(\left(\frac{1}{\mu\left(c_{\varepsilon}^{\alpha}\right)}-\frac{1}{\mu\left(c_{\varepsilon}\right)}\right) k \nabla p_{\varepsilon}^{\alpha}\right) \partial_{t} p_{\varepsilon}^{\alpha} d x
\end{aligned}
$$




$$
\begin{aligned}
& -2 \int_{\Omega} T_{\eta}^{\prime}\left(\left|k^{\frac{1}{2}} \nabla p_{\varepsilon}^{\alpha}\right|^{2}\right)\left(\frac{k}{\mu\left(c_{\varepsilon}\right)} \nabla p_{\varepsilon}^{\alpha} \cdot \nabla \phi\right) \partial_{t} p_{\varepsilon}^{\alpha} d x \\
& -2 \int_{\Omega} \phi T_{\eta}^{\prime \prime}\left(\left|k^{\frac{1}{2}} \nabla p_{\varepsilon}^{\alpha}\right|^{2}\right) \frac{k}{\mu\left(c_{\varepsilon}\right)}\left(\nabla p_{\varepsilon}^{\alpha} \cdot \nabla\left(\left|k^{\frac{1}{2}} \nabla p_{\varepsilon}^{\alpha}\right|^{2}\right)\right) \partial_{t} p_{\varepsilon}^{\alpha} d x .
\end{aligned}
$$

We now aim to obtain estimates, uniform with respect to $\varepsilon$, for the terms of the right-hand side of the latter relation. Using the Cauchy-Schwarz and Young inequalities, we can write for any $\delta>0$

$$
\begin{aligned}
\left|\int_{\Omega} \frac{\mu^{\prime}\left(c_{\varepsilon}\right)}{\mu^{2}\left(c_{\varepsilon}\right)} T_{\eta}\left(\left|k^{\frac{1}{2}} \nabla p_{\varepsilon}^{\alpha}\right|^{2}\right) \underline{u}_{\varepsilon} \cdot \nabla c_{\varepsilon}\right| \leq & \delta \int_{\Omega} \phi_{-} \alpha_{T}\left|\underline{u}_{\varepsilon}\right|\left|T_{\eta}\left(\left|k^{\frac{1}{2}} \nabla p_{\varepsilon}^{\alpha}\right|^{2}\right)\right|\left|\nabla c_{\varepsilon}\right|^{2} \\
& +\frac{C}{\delta} \int_{\Omega}\left|\underline{u}_{\varepsilon}\right|\left|T_{\eta}\left(\left|k^{\frac{1}{2}} \nabla p_{\varepsilon}^{\alpha}\right|^{2}\right)\right| \\
\leq & \delta \int_{\Omega} \phi_{-} \alpha_{T}\left|\underline{u}_{\varepsilon}\right|\left|T_{\eta}\left(\left|k^{\frac{1}{2}} \nabla p_{\varepsilon}^{\alpha}\right|^{2}\right)\right|\left|\nabla c_{\varepsilon}\right|^{2} \\
& +\frac{C}{\delta} \int_{\Omega}\left|\underline{u}_{\varepsilon}^{\alpha}\right|\left|T_{\eta}\left(\left|k^{\frac{1}{2}} \nabla p_{\varepsilon}^{\alpha}\right|^{2}\right)\right| \\
& +\frac{C}{\delta} \int_{\Omega}\left(\left|\underline{u}_{\varepsilon}\right|-\left|\underline{u}_{\varepsilon}^{\alpha}\right|\right)\left|T_{\eta}\left(\left|k^{\frac{1}{2}} \nabla p_{\varepsilon}^{\alpha}\right|^{2}\right)\right| \\
\leq & \delta \int_{\Omega} \phi_{-} \alpha_{T}\left|\underline{u}_{\varepsilon}\right|\left|T_{\eta}\left(\left|k^{\frac{1}{2}} \nabla p_{\varepsilon}^{\alpha}\right|^{2}\right)\right|\left|\nabla c_{\varepsilon}\right|^{2} \\
& +\frac{C(M+\eta)}{\delta} \int_{\Omega}\left|T_{\eta}\left(\left|k^{\frac{1}{2}} \nabla p_{\varepsilon}^{\alpha}\right|^{2}\right)\right| \\
& +\frac{C}{\delta} \int_{\Omega}\left(\left|\underline{u}_{\varepsilon}\right|-\left|\underline{u}_{\varepsilon}^{\alpha}\right|\right)\left|T_{\eta}\left(\left|k^{\frac{1}{2}} \nabla p_{\varepsilon}^{\alpha}\right|^{2}\right)\right| .
\end{aligned}
$$

The second term of the right-hand side of (3.4) writes

$$
\begin{aligned}
-\int_{\Omega} \frac{\mu^{\prime}\left(c_{\varepsilon}\right)}{\mu^{2}\left(c_{\varepsilon}\right)} \phi\left(\varepsilon+D_{p}\left(\underline{u}_{\varepsilon}\right)\right) \nabla c_{\varepsilon} \cdot T_{\eta}^{\prime}\left(\left|k^{\frac{1}{2}} \nabla p_{\varepsilon}^{\alpha}\right|^{2}\right) \nabla\left(\left|k^{\frac{1}{2}} \nabla p_{\varepsilon}^{\alpha}\right|^{2}\right) d x \\
=-2 \int_{\Omega} \frac{\mu^{\prime}\left(c_{\varepsilon}\right)}{\mu^{2}\left(c_{\varepsilon}\right)} \phi T_{\eta}^{\prime}\left(\left|k^{\frac{1}{2}} \nabla p_{\varepsilon}^{\alpha}\right|^{2}\right) \sum_{i=1}^{N}\left(\left(\sum_{j=1}^{N}\left(\varepsilon \delta_{i j}+D_{p}\left(\underline{u}_{\varepsilon}\right)_{i j}\right) \partial_{j} c_{\varepsilon}\right)\right. \\
\left.\quad \times\left(\sum_{j=1}^{N} \partial_{j} p_{\varepsilon}^{\alpha}\left(k \partial_{i j}^{2} p_{\varepsilon}^{\alpha}+\partial_{j} p_{\varepsilon}^{\alpha} \partial_{i} k\right)\right)\right) d x .
\end{aligned}
$$

Differentiating the Darcy law, we obtain $-k \partial_{i j}^{2} p_{\varepsilon}^{\alpha}-\partial_{j} p_{\varepsilon}^{\alpha} \partial_{i} k=\mu^{\prime}\left(c_{\varepsilon}^{\alpha}\right) \partial_{i} c_{\varepsilon}^{\alpha} \underline{u}_{\varepsilon, j}^{\alpha}+$ $\mu\left(c_{\varepsilon}^{\alpha}\right) \partial_{i} \underline{u}_{\varepsilon, j}^{\alpha}$. The first term of the right-hand side of the latter relation gives in (3.6)

$$
\begin{aligned}
& -2 \int_{\Omega} \frac{\mu^{\prime}\left(c_{\varepsilon}\right) \mu^{\prime}\left(c_{\varepsilon}^{\alpha}\right)}{\mu^{2}\left(c_{\varepsilon}\right) \mu\left(c_{\varepsilon}^{\alpha}\right)} \phi T_{\eta}^{\prime}\left(\left|k^{\frac{1}{2}} \nabla p_{\varepsilon}^{\alpha}\right|^{2}\right)\left|k^{\frac{1}{2}} \nabla p_{\varepsilon}^{\alpha}\right|^{2}\left(\varepsilon+D_{p}\left(\underline{u}_{\varepsilon}\right)\right) \nabla c_{\varepsilon} \cdot \nabla c_{\varepsilon} d x \\
& =-\int_{\Omega} \frac{2 \mu^{\prime 2}\left(c_{\varepsilon}\right)}{\mu^{3}\left(c_{\varepsilon}\right)} \phi T_{\eta}^{\prime}\left(\left|k^{\frac{1}{2}} \nabla p_{\varepsilon}^{\alpha}\right|^{2}\right)\left|k^{\frac{1}{2}} \nabla p_{\varepsilon}^{\alpha}\right|^{2}\left(\varepsilon+D_{p}\left(\underline{u}_{\varepsilon}\right)\right) \nabla c_{\varepsilon} \cdot \nabla c_{\varepsilon} d x
\end{aligned}
$$




$$
\begin{aligned}
& -\int_{\Omega} \frac{2 \mu^{\prime}\left(c_{\varepsilon}\right) \mu^{\prime}\left(c_{\varepsilon}^{\alpha}\right)}{\mu^{2}\left(c_{\varepsilon}\right)}\left(\frac{\phi}{\mu\left(c_{\varepsilon}^{\alpha}\right)}-\frac{\phi}{\mu\left(c_{\varepsilon}\right)}\right) \\
& \times T_{\eta}^{\prime}\left(\left|k^{\frac{1}{2}} \nabla p_{\varepsilon}^{\alpha}\right|^{2}\right)\left|k^{\frac{1}{2}} \nabla p_{\varepsilon}^{\alpha}\right|^{2}\left(\varepsilon+D_{p}\left(\underline{u}_{\varepsilon}\right)\right) \\
& \times \nabla c_{\varepsilon} \cdot \nabla c_{\varepsilon} d x-\int_{\Omega} \frac{2 \mu^{\prime 2}\left(c_{\varepsilon}\right)}{\mu^{3}\left(c_{\varepsilon}\right)}\left(\mu^{\prime}\left(c_{\varepsilon}^{\alpha}\right)-\mu^{\prime}\left(c_{\varepsilon}\right)\right) \phi \\
& \times T_{\eta}^{\prime}\left(\left|k^{\frac{1}{2}} \nabla p_{\varepsilon}^{\alpha}\right|^{2}\right)\left|k^{\frac{1}{2}} \nabla p_{\varepsilon}^{\alpha}\right|^{2}\left(\varepsilon+D_{p}\left(\underline{u}_{\varepsilon}\right)\right) \nabla c_{\varepsilon} \cdot \nabla c_{\varepsilon} d x .
\end{aligned}
$$

With the second term in the expression of $-k \partial_{i j}^{2} p_{\varepsilon}^{\alpha}-\partial_{j} p_{\varepsilon}^{\alpha} \partial_{i} k$ in (3.6), the CauchySchwarz inequality and Assumption (1.5) for $k$, we get

$$
\begin{aligned}
\mid 2 \int_{\Omega} \frac{\mu\left(c_{\varepsilon}^{\alpha}\right) \mu^{\prime}\left(c_{\varepsilon}\right)}{\mu^{2}\left(c_{\varepsilon}\right)} \phi T_{\eta}^{\prime}\left(\left|k^{\frac{1}{2}} \nabla p_{\varepsilon}^{\alpha}\right|^{2}\right) \sum_{i=1}^{N}\left(\left(\sum_{j=1}^{N}\left(\varepsilon \delta_{i j}+D_{p}\left(\underline{u}_{\varepsilon}\right)_{i j}\right) \partial_{j} c_{\varepsilon}\right)\right. \\
\left.\quad \times\left(\sum_{j=1}^{N} \partial_{j} p_{\varepsilon}^{\alpha} \partial_{i} \underline{u}_{\varepsilon, j}^{\alpha}\right)\right) d x \mid \\
\leq C \sum_{i, j=1}^{N}\left(\int_{\Omega}\left|T_{\eta}^{\prime}\left(\left|k^{\frac{1}{2}} \nabla p_{\varepsilon}^{\alpha}\right|^{2}\right)\right|\left|\partial_{i} \underline{u}_{\varepsilon, j}^{\alpha}\right|^{2} d x\right)^{1 / 2} \\
\times\left(\int_{\Omega}\left|T_{\eta}^{\prime}\left(\left|k^{\frac{1}{2}} \nabla p_{\varepsilon}^{\alpha}\right|^{2}\right)\right|\left(\varepsilon^{2}+\alpha_{L}\left|\underline{u}_{\varepsilon}\right|^{2}\right)\left|\nabla p_{\varepsilon}^{\alpha}\right|^{2}|\nabla c|^{2} d x\right)^{1 / 2} .
\end{aligned}
$$

Introducing $\left|\underline{u}_{\varepsilon}^{\alpha}\right|$ in the latter relation and using the definition of $T_{\eta}$, we obtain

$$
\begin{aligned}
& \mid 2 \int_{\Omega} \frac{\mu\left(c_{\varepsilon}^{\alpha}\right) \mu^{\prime}\left(c_{\varepsilon}\right)}{\mu^{2}\left(c_{\varepsilon}\right)} \phi T_{\eta}^{\prime}\left(\left|k^{\frac{1}{2}} \nabla p_{\varepsilon}^{\alpha}\right|^{2}\right) \sum_{i=1}^{N}\left(\left(\sum_{j=1}^{N}\left(\varepsilon \delta_{i j}+D_{p}\left(\underline{u}_{\varepsilon}\right)_{i j}\right) \partial_{j} c_{\varepsilon}\right)\right. \\
& \left.\quad \times\left(\sum_{j=1}^{N} \partial_{j} p_{\varepsilon}^{\alpha} \partial_{i} \underline{u}_{\varepsilon, j}^{\alpha}\right)\right) \mid \leq C \sum_{i, j=1}^{N}\left(\int_{\Omega}\left|T_{\eta}^{\prime}\left(\left|k^{\frac{1}{2}} \nabla p_{\varepsilon}^{\alpha}\right|^{2}\right)\right|\left|\partial_{i} \underline{u}_{\varepsilon, j}^{\alpha}\right|^{2}\right)^{1 / 2} \\
& \quad \times\left(\int_{\Omega}\left|T_{\eta}^{\prime}\left(\left|k^{\frac{1}{2}} \nabla p_{\varepsilon}^{\alpha}\right|^{2}\right)\right|\left(\varepsilon^{2}+\alpha_{L}\left|\underline{u}_{\varepsilon}\right|\left|\underline{u}_{\varepsilon}^{\alpha}\right|+\alpha_{L}\left|\underline{u}_{\varepsilon}\right|\left(\left|\underline{u}_{\varepsilon}\right|-\left|\underline{u}_{\varepsilon}^{\alpha}\right|\right)\right)\left|\nabla p_{\varepsilon}^{\alpha}\right|^{2}\left|\nabla c_{\varepsilon}\right|^{2}\right)^{1 / 2} \\
& \leq \sum_{i, j=1}^{N} C(M+\eta)^{3 / 2}\left(\int_{\Omega}\left(\varepsilon^{2}+\left|\underline{u}_{\varepsilon}\right|\right)\left|\nabla c_{\varepsilon}\right|^{2}\right)^{1 / 2}\left(\int_{\Omega}\left|T_{\eta}^{\prime}\left(\left|k^{\frac{1}{2}} \nabla p_{\varepsilon}^{\alpha}\right|^{2}\right)\right|\left|\partial_{i} \underline{u}_{\varepsilon, j}^{\alpha}\right|^{2}\right)^{1 / 2} \\
& \quad+\sum_{i, j=1}^{N} C\left(\int_{\Omega}\left|T_{\eta}^{\prime}\left(\left|k^{\frac{1}{2}} \nabla p_{\varepsilon}^{\alpha}\right|^{2}\right)\right|\left|\underline{u}_{\varepsilon}\right|\left(\left|\underline{u}_{\varepsilon}\right|-\left|\underline{u}_{\varepsilon}^{\alpha}\right|\right)\left|\nabla p_{\varepsilon}^{\alpha}\right|^{2}\left|\nabla c_{\varepsilon}\right|^{2}\right)^{1 / 2} \\
& \quad \times\left(\int_{\Omega}\left|T_{\eta}^{\prime}\left(\left|k^{\frac{1}{2}} \nabla p_{\varepsilon}^{\alpha}\right|^{2}\right)\right|\left|\partial_{i} \underline{u}_{\varepsilon, j}^{\alpha}\right|^{2}\right)^{1 / 2}
\end{aligned}
$$


and then

$$
\begin{aligned}
& \mid 2 \int_{\Omega} \frac{\mu\left(c_{\varepsilon}^{\alpha}\right) \mu^{\prime}\left(c_{\varepsilon}\right)}{\mu^{2}\left(c_{\varepsilon}\right)} \phi T_{\eta}^{\prime}\left(\left|k^{\frac{1}{2}} \nabla p_{\varepsilon}^{\alpha}\right|^{2}\right) \sum_{i=1}^{N}\left(\left(\sum_{j=1}^{N}\left(\varepsilon \delta_{i j}+D_{p}\left(\underline{u}_{\varepsilon}\right)_{i j}\right) \partial_{j} c_{\varepsilon}\right)\right. \\
& \left.\quad \times\left(\sum_{j=1}^{N} \partial_{j} p_{\varepsilon}^{\alpha} \partial_{i} \underline{u}_{\varepsilon, j}^{\alpha}\right)\right) \mid \leq \sum_{i, j=1}^{N} C(t) \frac{(M+\eta)^{3 / 2}}{m} \\
& \quad \times\left(\int_{\Omega \cap B_{m, M}}\left|T_{\eta}\left(\left|k^{\frac{1}{2}} \nabla p_{\varepsilon}^{\alpha}\right|^{2}\right)\right|\right)^{1 / 2}\left(\int_{\Omega}\left|T_{\eta}^{\prime}\left(\left|k^{\frac{1}{2}} \nabla p_{\varepsilon}^{\alpha}\right|^{2}\right)\right|\left|\partial_{i} \underline{u}_{\varepsilon, j}^{\alpha}\right|^{2}\right)^{1 / 2} \\
& \quad+\sum_{i, j=1}^{N} C\left(\int_{\Omega}\left|T_{\eta}^{\prime}\left(\left|k^{\frac{1}{2}} \nabla p_{\varepsilon}^{\alpha}\right|^{2}\right)\right|\left|\partial_{i} \underline{u}_{\varepsilon, j}^{\alpha}\right|^{2}\right)^{1 / 2} \\
& \quad \times\left(\int_{\Omega}\left|T_{\eta}^{\prime}\left(\left|k^{\frac{1}{2}} \nabla p_{\varepsilon}^{\alpha}\right|^{2}\right)\right|\left|\underline{u}_{\varepsilon}\right|\left(\left|\underline{u}_{\varepsilon}\right|-\left|\underline{u}_{\varepsilon}^{\alpha}\right|\right)\left|\nabla p_{\varepsilon}^{\alpha}\right|^{2}\left|\nabla c_{\varepsilon}\right|^{2}\right)^{1 / 2},
\end{aligned}
$$

where the quantity $C(t)$ is uniformly bounded in $L^{2}(0, T)$ thanks to Lemma 2 . We have denoted by $B_{m, M}$ the set of points of $\Omega$ such that $m \leq\left|k^{1 / 2} \nabla p_{\varepsilon}^{\alpha}(\cdot, t)\right| \leq M$. Now, for any given real $\delta>0$, let $f_{\delta}\left(\left|k^{\frac{1}{2}} \nabla p_{\varepsilon}^{\alpha}\right|^{2}\right)=\sqrt{\left.T_{\eta}^{\prime}\left(\left|k^{\frac{1}{2}} \nabla p_{\varepsilon}^{\alpha}\right|^{2}\right)+\delta\right)}$. Integrating by parts, one easily checks that

$$
\begin{aligned}
\sum_{i, j=1}^{N} \int_{\Omega} f_{\delta}\left(\left|k^{\frac{1}{2}} \nabla p_{\varepsilon}^{\alpha}\right|^{2}\right)\left|\partial_{i} \underline{u}_{\varepsilon, j}^{\alpha}\right|^{2} d x= & \int_{\Omega} f_{\delta}\left(\left|k^{\frac{1}{2}} \nabla p_{\varepsilon}^{\alpha}\right|^{2}\right)\left(\operatorname{div}\left(\underline{u}_{\varepsilon}^{\alpha}\right)^{2}+\left|\operatorname{curl}\left(\underline{u}_{\varepsilon}^{\alpha}\right)\right|^{2}\right) d x \\
& +2 \sum_{i \neq j} \int_{\Omega} f_{\delta}^{\prime}\left(\left|k^{\frac{1}{2}} \nabla p_{\varepsilon}^{\alpha}\right|^{2}\right) \underline{u}_{\varepsilon, j}^{\alpha} \\
& \times\left(-\partial_{i} \underline{u}_{\varepsilon, i}^{\alpha} \partial_{j}\left(\left|k^{\frac{1}{2}} \nabla p_{\varepsilon}^{\alpha}\right|^{2}\right)\right. \\
& \left.+\partial_{j} \underline{u}_{\varepsilon, i}^{\alpha} \partial_{i}\left(\left|k^{\frac{1}{2}} \nabla p_{\varepsilon}^{\alpha}\right|^{2}\right)\right) d x .
\end{aligned}
$$

Letting $\delta \rightarrow 0$, we obtain

$$
\begin{aligned}
\sum_{i, j=1}^{N} \int_{\Omega} \mid T_{\eta}^{\prime}\left(\mid k^{\frac{1}{2}} \nabla\right. & \left.\left.p_{\varepsilon}^{\alpha}\right|^{2}\right)\left.|| \partial_{i} \underline{u}_{\varepsilon, j}^{\alpha}\right|^{2} d x=\int_{\Omega}\left|T_{\eta}^{\prime}\left(\left|k^{\frac{1}{2}} \nabla p_{\varepsilon}^{\alpha}\right|^{2}\right)\right|\left(\operatorname{div}\left(\underline{u}_{\varepsilon}^{\alpha}\right)^{2}\right. \\
& \left.+\left|\operatorname{curl}\left(\underline{u}_{\varepsilon}^{\alpha}\right)\right|^{2}\right) d x \\
& +2 \sum_{i \neq j} \int_{\Omega} \operatorname{sign}\left(T_{\eta}^{\prime}\left(\left|k^{\frac{1}{2}} \nabla p_{\varepsilon}^{\alpha}\right|^{2}\right)\right) T_{\eta}^{\prime \prime}\left(\left|k^{\frac{1}{2}} \nabla p_{\varepsilon}^{\alpha}\right|^{2}\right) \underline{u}_{\varepsilon, j}^{\alpha} \\
& \times\left(-\partial_{i} \underline{u}_{\varepsilon, i}^{\alpha} \partial_{j}\left(\left|k^{\frac{1}{2}} \nabla p_{\varepsilon}^{\alpha}\right|^{2}\right)\right. \\
& \left.+\partial_{j} \underline{u}_{\varepsilon, i}^{\alpha} \partial_{i}\left(\left|k^{\frac{1}{2}} \nabla p_{\varepsilon}^{\alpha}\right|^{2}\right)\right) d x .
\end{aligned}
$$

We denote $I_{\eta}$ the second term of the right-hand side of (3.9). We note that

$$
\lim _{\eta \rightarrow 0} I_{\eta}=0
$$


because the regularities $T_{\eta}^{\prime \prime}\left(\left|k^{\frac{1}{2}} \nabla p_{\varepsilon}^{\alpha}\right|^{2}\right)\left|\underline{u}_{\varepsilon}^{\alpha}\right|^{2} \in L^{\infty}\left(\Omega_{T}\right), p_{\varepsilon}^{\alpha} \in L^{2}\left(0, T ; W^{2,2}(\Omega)\right)$ and $k \in W^{1, \infty}(\Omega)$ imply that the integrated function belongs to $L^{1}\left(\Omega_{T}\right)$. We bear in mind that $\operatorname{div}\left(\underline{u}_{\varepsilon}^{\alpha}\right)=-\partial_{t} p_{\varepsilon}^{\alpha}$ and $\left|\operatorname{curl}\left(u_{\varepsilon}^{\alpha}\right)\right| \leq C\left(\left|\nabla c_{\varepsilon}^{\alpha}\right|+1\right)\left|\nabla p_{\varepsilon}^{\alpha}\right|$. Then, using (3.9) in (3.8) and the Young inequality, we get for any $\delta>0$

$$
\begin{aligned}
& \mid 2 \int_{\Omega} \frac{\mu\left(c_{\varepsilon}^{\alpha}\right) \mu^{\prime}\left(c_{\varepsilon}\right)}{\mu^{2}\left(c_{\varepsilon}\right)} \phi T_{\eta}^{\prime}\left(\left|k^{\frac{1}{2}} \nabla p_{\varepsilon}^{\alpha}\right|^{2}\right) \sum_{i=1}^{N}\left(\left(\sum_{j=1}^{N}\left(\varepsilon \delta_{i j}+D_{p}\left(\underline{u}_{\varepsilon}\right)_{i j}\right) \partial_{j} c_{\varepsilon}\right)\right. \\
& \left.\quad \times\left(\sum_{j=1}^{N} \partial_{j} p_{\varepsilon}^{\alpha} \partial_{i} \underline{u}_{\varepsilon, j}^{\alpha}\right)\right) d x \mid \\
& \leq C(t) \frac{(M+\eta)^{3 / 2}}{m}\left(\int_{\Omega \cap B_{m, M}}\left|T_{\eta}\left(\left|k^{\frac{1}{2}} \nabla p_{\varepsilon}^{\alpha}\right|^{2}\right)\right| d x\right)^{1 / 2} I_{\eta} \\
& \quad+C\left(\int_{\Omega}\left|T_{\eta}^{\prime}\left(\left|k^{\frac{1}{2}} \nabla p_{\varepsilon}^{\alpha}\right|^{2}\right)\right|\left|\underline{u}_{\varepsilon}\right|\left(\left|\underline{u}_{\varepsilon}\right|-\left|\underline{u}_{\varepsilon}^{\alpha}\right|\right)\left|\nabla p_{\varepsilon}^{\alpha}\right|^{2}\left|\nabla c_{\varepsilon}\right|^{2} d x\right)^{1 / 2} I_{\eta} \\
& \quad+\frac{C(t)(M+\eta)^{3}}{m^{2} \delta} \int_{\Omega \cap B_{m, M}}\left|T_{\eta}\left(\left|k^{\frac{1}{2}} \nabla p_{\varepsilon}^{\alpha}\right|^{2}\right)\right| d x \\
& \quad+\frac{C}{\delta} \int_{\Omega}\left|T_{\eta}^{\prime}\left(\left|k^{\frac{1}{2}} \nabla p_{\varepsilon}^{\alpha}\right|^{2}\right)\right|\left|\underline{u}_{\varepsilon}\right|\left(\left|\underline{u}_{\varepsilon}\right|-\left|\underline{u}_{\varepsilon}^{\alpha}\right|\right)\left|\nabla p_{\varepsilon}^{\alpha}\right|^{2}\left|\nabla c_{\varepsilon}\right|^{2} d x \\
& \quad+\delta \int_{\Omega}\left|T_{\eta}^{\prime}\left(\left|k^{\frac{1}{2}} \nabla p_{\varepsilon}^{\alpha}\right|^{2}\right)\right|\left|\partial_{t} p_{\varepsilon}^{\alpha}\right|^{2} d x+C(t) \frac{(M+\eta)^{3 / 2}}{m} \\
& \quad\left(\int_{\Omega \cap B_{m, M}}\left|T_{\eta}\left(\left|k^{\frac{1}{2}} \nabla p_{\varepsilon}^{\alpha}\right|^{2}\right)\right| d x\right)^{1 / 2}\left(\int_{\Omega}\left|T_{\eta}^{\prime}\left(\left|k^{\frac{1}{2}} \nabla p_{\varepsilon}^{\alpha}\right|^{2}\right)\right|\left(\left|\nabla c_{\varepsilon}^{\alpha}\right|+1\right)^{2}\left|\nabla p_{\varepsilon}^{\alpha}\right|^{2} d x\right)^{1 / 2} \\
& \quad+C\left(\int_{\Omega}\left|T_{\eta}^{\prime}\left(\left|k^{\frac{1}{2}} \nabla p_{\varepsilon}^{\alpha}\right|^{2}\right)\right|\left|\underline{u}_{\varepsilon}\right|\left(\left|\underline{u}_{\varepsilon}\right|-\left|\underline{u}_{\varepsilon}^{\alpha}\right|\right)\left|\nabla p_{\varepsilon}^{\alpha}\right|^{2}\left|\nabla c_{\varepsilon}\right|^{2} d x\right)^{1 / 2} \\
& \quad \times\left(\int_{\Omega}\left|T_{\eta}^{\prime}\left(\left|k^{\frac{1}{2}} \nabla p_{\varepsilon}^{\alpha}\right|^{2}\right)\right|\left(\left|\nabla c_{\varepsilon}^{\alpha}\right|+1\right)^{2}\left|\nabla p_{\varepsilon}^{\alpha}\right|^{2} d x\right)^{1 / 2} \cdot
\end{aligned}
$$

We have

$$
\begin{aligned}
\frac{(M+\eta)^{3 / 2}}{m}\left(\int_{\Omega \cap B_{m, M}} \mid\right. & \left.T_{\eta}\left(\left|k^{\frac{1}{2}} \nabla p_{\varepsilon}^{\alpha}\right|^{2}\right) \mid\right)^{1 / 2} \\
& \times\left(\int_{\Omega}\left|T_{\eta}^{\prime}\left(\left|k^{\frac{1}{2}} \nabla p_{\varepsilon}^{\alpha}\right|^{2}\right)\right|\left(\left|\nabla c_{\varepsilon}^{\alpha}\right|+1\right)^{2}\left|\nabla p_{\varepsilon}^{\alpha}\right|^{2}\right)^{1 / 2} \\
\leq & C\left(\frac{M+\eta}{m}\right)^{3 / 2}\left(\int_{\Omega \cap B_{m, M}}\left|T_{\eta}\left(\left|k^{\frac{1}{2}} \nabla p_{\varepsilon}^{\alpha}\right|^{2}\right)\right| d x\right)^{1 / 2} \\
& \times\left(\int_{\Omega}\left|T_{\eta}^{\prime}\left(\left|k^{\frac{1}{2}} \nabla p_{\varepsilon}^{\alpha}\right|^{2}\right)\right|\left|\underline{u}_{\varepsilon}^{\alpha}\right|\left|\nabla p_{\varepsilon}^{\alpha}\right|^{2}\left(\left|\nabla c_{\varepsilon}^{\alpha}\right|+1\right)^{2} d x\right)^{1 / 2}
\end{aligned}
$$


and using the Young inequality

$$
\begin{aligned}
& \frac{(M+\eta)^{3 / 2}}{m}\left(\int_{\Omega \cap B_{m, M}}\left|T_{\eta}\left(\left|k^{\frac{1}{2}} \nabla p_{\varepsilon}^{\alpha}\right|^{2}\right)\right|\right)^{1 / 2}\left(\int_{\Omega}\left|T_{\eta}^{\prime}\left(\left|k^{\frac{1}{2}} \nabla p_{\varepsilon}^{\alpha}\right|^{2}\right)\right|\left(\left|\nabla c_{\varepsilon}^{\alpha}\right|+1\right)^{2}\right. \\
&\left.\quad \times\left|\nabla p_{\varepsilon}^{\alpha}\right|^{2}\right)^{1 / 2} \leq \delta C \int_{\Omega} \phi_{-} \alpha_{T}\left|T_{\eta}^{\prime}\left(\left|k^{\frac{1}{2}} \nabla p_{\varepsilon}^{\alpha}\right|^{2}\right)\right|\left|\underline{u}_{\varepsilon}\right|\left|\nabla p_{\varepsilon}^{\alpha}\right|^{2}\left|\nabla c_{\varepsilon}\right|^{2} d x \\
& \\
& \quad+C \frac{(M+\eta)^{3}}{\delta m^{3}} \int_{\Omega \cap B_{m}, M}\left|T_{\eta}\left(\left|k^{\frac{1}{2}} \nabla p_{\varepsilon}^{\alpha}\right|^{2}\right)\right| d x \\
&+C(M+\eta) \int_{\Omega}\left|T_{\eta}\left(\left|k^{\frac{1}{2}} \nabla p_{\varepsilon}^{\alpha}\right|^{2}\right)\right| d x \\
&+\delta C \int_{\Omega}\left|T_{\eta}^{\prime}\left(\left|k^{\frac{1}{2}} \nabla p_{\varepsilon}^{\alpha}\right|^{2}\right)\right|\left(\left|\underline{u}_{\varepsilon}^{\alpha}\right|-\left|\underline{u}_{\varepsilon}\right|\right)\left|\nabla p_{\varepsilon}^{\alpha}\right|^{2}\left|\nabla c_{\varepsilon}^{\alpha}\right|^{2} d x \\
&+\left.\delta C \int_{\Omega}\left|T_{\eta}^{\prime}\left(\left|k^{\frac{1}{2}} \nabla p_{\varepsilon}^{\alpha}\right|^{2}\right)\right|\left|\underline{u}_{\varepsilon}^{\alpha}\right|\left|\nabla p_{\varepsilon}^{\alpha}\right|^{2}|| \nabla c_{\varepsilon}^{\alpha}\right|^{2}-\left|\nabla c_{\varepsilon}\right|^{2} \mid d x
\end{aligned}
$$

for any $\delta>0$. We thus obtain in (3.10)

$$
\begin{aligned}
& \mid 2 \int_{\Omega} \frac{\mu\left(c_{\varepsilon}^{\alpha}\right) \mu^{\prime}\left(c_{\varepsilon}\right)}{\mu^{2}\left(c_{\varepsilon}\right)} \phi T_{\eta}^{\prime}\left(\left|k^{\frac{1}{2}} \nabla p_{\varepsilon}^{\alpha}\right|^{2}\right) \sum_{i=1}^{N}\left(\left(\sum_{j=1}^{N}\left(\varepsilon \delta_{i j}+D_{p}\left(\underline{u}_{\varepsilon}\right)_{i j}\right) \partial_{j} c_{\varepsilon}\right)\right. \\
& \left.\quad \times\left(\sum_{j=1}^{N} \partial_{j} p_{\varepsilon}^{\alpha} \partial_{i} \underline{u}_{\varepsilon, j}^{\alpha}\right)\right) \mid \leq C(t) \frac{(M+\eta)^{3 / 2}}{m}\left(\int_{\Omega \cap B_{m, M}}\left|T_{\eta}\left(\left|k^{\frac{1}{2}} \nabla p_{\varepsilon}^{\alpha}\right|^{2}\right)\right|\right)^{1 / 2} I_{\eta} \\
& +C\left(\int_{\Omega}\left|T_{\eta}^{\prime}\left(\left|k^{\frac{1}{2}} \nabla p_{\varepsilon}^{\alpha}\right|^{2}\right)\right|\left|\underline{u}_{\varepsilon}\right||| \underline{u}_{\varepsilon}|-| \underline{u}_{\varepsilon}^{\alpha}||\left|\nabla p_{\varepsilon}^{\alpha}\right|^{2}\left|\nabla c_{\varepsilon}\right|^{2}\right)^{1 / 2} I_{\eta} \\
& \quad+C(t) \frac{(M+\eta)^{3}}{m^{2} \delta} \int_{\Omega \cap B_{m, M}}\left|T_{\eta}\left(\left|k^{\frac{1}{2}} \nabla p_{\varepsilon}^{\alpha}\right|^{2}\right)\right|+C(t)(M+\eta) \int_{\Omega}\left|T_{\eta}\left(\left|k^{\frac{1}{2}} \nabla p_{\varepsilon}^{\alpha}\right|^{2}\right)\right| \\
& \quad+\frac{C}{\delta} \int_{\Omega}\left|T_{\eta}^{\prime}\left(\left|k^{\frac{1}{2}} \nabla p_{\varepsilon}^{\alpha}\right|^{2}\right)\right|\left|\underline{u}_{\varepsilon}\right|\left(\left|\underline{u}_{\varepsilon}\right|-\left|\underline{u}_{\varepsilon}^{\alpha}\right|\right)\left|\nabla p_{\varepsilon}^{\alpha}\right|^{2}\left|\nabla c_{\varepsilon}\right|^{2} \\
& +\delta \int_{\Omega}\left|T_{\eta}^{\prime}\left(\left|k^{\frac{1}{2}} \nabla p_{\varepsilon}^{\alpha}\right|^{2}\right)\right|\left|\partial_{t} p_{\varepsilon}^{\alpha}\right|^{2}+\delta \int_{\Omega} \phi \alpha_{T}\left|T_{\eta}^{\prime}\left(\left|k^{\frac{1}{2}} \nabla p_{\varepsilon}^{\alpha}\right|^{2}\right)\right|\left|\underline{u}_{\varepsilon}\right|\left|\nabla p_{\varepsilon}^{\alpha}\right|^{2}\left|\nabla c_{\varepsilon}\right|^{2} \\
& +C(t)(M+\eta)^{1 / 2}\left(\int_{\Omega}\left|T_{\eta}^{\prime}\left(\left|k^{\frac{1}{2}} \nabla p_{\varepsilon}^{\alpha}\right|^{2}\right)\right|\left|\underline{u}_{\varepsilon}\right||| \underline{u}_{\varepsilon}|-| \underline{u}_{\varepsilon}^{\alpha}||\left|\nabla p_{\varepsilon}^{\alpha}\right|^{2}\left|\nabla c_{\varepsilon}\right|^{2}\right)^{1 / 2} \\
& +C(t) \delta \int_{\Omega}\left|T_{\eta}^{\prime}\left(\left|k^{\frac{1}{2}} \nabla p_{\varepsilon}^{\alpha}\right|^{2}\right)\right||| \underline{u}_{\varepsilon}^{\alpha}|-| \underline{u}_{\varepsilon}||\left|\nabla p_{\varepsilon}^{\alpha}\right|^{2}\left|\nabla c_{\varepsilon}^{\alpha}\right|^{2} .
\end{aligned}
$$


Another term of (3.4) is estimated as follows. We write

$$
\begin{aligned}
\int_{\Omega} \phi & T_{\eta}^{\prime}\left(\left|k^{\frac{1}{2}} \nabla p_{\varepsilon}^{\alpha}\right|^{2}\right) \operatorname{div}\left(\left(\frac{1}{\mu\left(c_{\varepsilon}^{\alpha}\right)}-\frac{1}{\mu\left(c_{\varepsilon}\right)}\right) k \nabla p_{\varepsilon}^{\alpha}\right) \partial_{t} p_{\varepsilon}^{\alpha} d x \\
= & \int_{\Omega} \phi T_{\eta}^{\prime}\left(\left|k^{\frac{1}{2}} \nabla p_{\varepsilon}^{\alpha}\right|^{2}\right)\left(\left(-\frac{\mu^{\prime}\left(c_{\varepsilon}^{\alpha}\right)}{\mu^{2}\left(c_{\varepsilon}^{\alpha}\right)} \nabla c_{\varepsilon}^{\alpha}+\frac{\mu^{\prime}\left(c_{\varepsilon}\right)}{\mu^{2}\left(c_{\varepsilon}\right)} \nabla c_{\varepsilon}\right) \cdot k \nabla p_{\varepsilon}^{\alpha}\right) \partial_{t} p_{\varepsilon}^{\alpha} d x \\
& +\int_{\Omega} \phi T_{\eta}^{\prime}\left(\left|k^{\frac{1}{2}} \nabla p_{\varepsilon}^{\alpha}\right|^{2}\right)\left(\frac{1}{\mu\left(c_{\varepsilon}^{\alpha}\right)}-\frac{1}{\mu\left(c_{\varepsilon}\right)}\right) \operatorname{div}\left(k \nabla p_{\varepsilon}^{\alpha}\right) \partial_{t} p_{\varepsilon}^{\alpha} d x
\end{aligned}
$$

Finally, using the Cauchy-Schwarz and Young inequalities and the regularity of the porosity $\phi \in W^{1, \infty}(\Omega)$, we get for any $\delta>0$

$$
\begin{aligned}
& \left|\int_{\Omega} T_{\eta}^{\prime}\left(\left|k^{\frac{1}{2}} \nabla p_{\varepsilon}^{\alpha}\right|^{2}\right)\left(\frac{k}{\mu\left(c_{\varepsilon}\right)} \nabla p_{\varepsilon}^{\alpha} \cdot \nabla \phi\right) \partial_{t} p_{\varepsilon}^{\alpha} d x\right| \\
& \quad \leq \delta \int_{\Omega}\left|T_{\eta}^{\prime}\left(\left|k^{\frac{1}{2}} \nabla p_{\varepsilon}^{\alpha}\right|^{2}\right)\right|\left|\partial_{t} p_{\varepsilon}^{\alpha}\right|^{2} d x+\frac{C}{\delta} \int_{\Omega}\left|T_{\eta}^{\prime}\left(\left|k^{\frac{1}{2}} \nabla p_{\varepsilon}^{\alpha}\right|^{2}\right)\right|\left|\nabla p_{\varepsilon}^{\alpha}\right|^{2} d x .
\end{aligned}
$$

We now collect Estimates (3.5), (3.7), (3.11)-(3.13) in (3.4). Denoting by $C$ a generic quantity belonging to $L^{1}(0, T)$, we obtain for any $\delta>0$

$$
\begin{aligned}
\frac{d}{d t} \int_{\Omega} & \frac{\phi}{\mu\left(c_{\varepsilon}\right)} T_{\eta}\left(\left|k^{\frac{1}{2}} \nabla p_{\varepsilon}^{\alpha}\right|^{2}\right) d x+\int_{\Omega} T_{\eta}^{\prime}\left(\left|k^{\frac{1}{2}} \nabla p_{\varepsilon}^{\alpha}\right|^{2}\right)(2 \phi-2 \delta)\left|\partial_{t} p_{\varepsilon}^{\alpha}\right|^{2} d x \\
& +\int_{\Omega}\left(\frac{\mu^{\prime \prime} \mu}{\mu^{3}}\right)\left(c_{\varepsilon}\right) T_{\eta}\left(\left|k^{\frac{1}{2}} \nabla p_{\varepsilon}^{\alpha}\right|^{2}\right) \phi_{-}(1-2 \delta)\left(\varepsilon+\alpha_{T}\left|\underline{u}_{\varepsilon}\right|\right)\left|\nabla c_{\varepsilon}\right|^{2} d x \\
\leq & \frac{C(t)}{\delta}(M+\eta) \int_{\Omega} T_{\eta}\left(\left|k^{\frac{1}{2}} \nabla p_{\varepsilon}^{\alpha}\right|^{2}\right) d x+\frac{C(t)}{\delta} \frac{(M+\eta)^{3}}{m^{2}} \int_{\Omega \cap B_{m, M}} T_{\eta}\left(\left|k^{\frac{1}{2}} \nabla p_{\varepsilon}^{\alpha}\right|^{2}\right) d x \\
& +\frac{C(M+\eta)}{\delta}+\frac{C}{\delta} \int_{\Omega}\left|T_{\eta}^{\prime}\left(\left|k^{\frac{1}{2}} \nabla p_{\varepsilon}^{\alpha}\right|^{2}\right)\right|\left|\nabla p_{\varepsilon}^{\alpha}\right|^{2} d x \\
& +\frac{C}{\delta} \int_{\Omega}|| \underline{u}_{\varepsilon}|-| \underline{u}_{\varepsilon}^{\alpha}|| T_{\eta}\left(\left|k^{\frac{1}{2}} \nabla p_{\varepsilon}^{\alpha}\right|^{2}\right) \\
& +C \int_{\Omega}\left|\frac{1}{\mu\left(c_{\varepsilon}^{\alpha}\right)}-\frac{1}{\mu\left(c_{\varepsilon}\right)}\right|\left|T_{\eta}^{\prime}\left(\left|k^{\frac{1}{2}} \nabla p_{\varepsilon}^{\alpha}\right|^{2}\right)\right|\left(\varepsilon+\alpha \underline{u}_{\varepsilon} \mid\right)\left|\nabla p_{\varepsilon}^{\alpha}\right|^{2}\left|\nabla c_{\varepsilon}\right|^{2} d x \\
& +C \int_{\Omega}\left|\mu^{\prime}\left(c_{\varepsilon}^{\alpha}\right)-\mu^{\prime}\left(c_{\varepsilon}\right)\right|\left|T_{\eta}^{\prime}\left(\left|k^{\frac{1}{2}} \nabla p_{\varepsilon}^{\alpha}\right|^{2}\right)\right|\left|k^{\frac{1}{2}} \nabla p_{\varepsilon}^{\alpha}\right|^{2}\left(\varepsilon+D_{p}\left(\underline{u}_{\varepsilon}\right)\right) \nabla c_{\varepsilon} \cdot \nabla c_{\varepsilon} d x \\
& +\frac{C}{\delta} \int_{\Omega}\left|T_{\eta}^{\prime}\left(\left|k^{\frac{1}{2}} \nabla p_{\varepsilon}^{\alpha}\right|^{2}\right)\right|\left|\underline{u}_{\varepsilon}\right||| \underline{u}_{\varepsilon}|-| \underline{u}_{\varepsilon}^{\alpha}||\left|\nabla p_{\varepsilon}^{\alpha}\right|^{2}\left(\left|\nabla c_{\varepsilon}\right|^{2}+\left|\nabla c_{\varepsilon}^{\alpha}\right|^{2}\right) d x \\
& +\int_{\Omega} \phi\left|T_{\eta}^{\prime}\left(\left|k^{\frac{1}{2}} \nabla p_{\varepsilon}^{\alpha}\right|^{2}\right)\right|\left|\left(-\frac{\mu^{\prime}\left(c_{\varepsilon}^{\alpha}\right)}{\mu\left(c_{\varepsilon}^{\alpha}\right)} \nabla c_{\varepsilon}^{\alpha}+\frac{\mu^{\prime}\left(c_{\varepsilon}\right)}{\mu\left(c_{\varepsilon}\right)} \nabla c_{\varepsilon}\right) \cdot k \nabla p_{\varepsilon}^{\alpha}\right|\left|\partial_{t} p_{\varepsilon}^{\alpha}\right| d x \\
& +\int_{\Omega} \phi\left|T_{\eta}^{\prime}\left(\left|k^{\frac{1}{2}} \nabla p_{\varepsilon}^{\alpha}\right|^{2}\right)\right|\left|\frac{1}{\mu\left(c_{\varepsilon}^{\alpha}\right)}-\frac{1}{\mu\left(c_{\varepsilon}\right)}\right|\left|\operatorname{div}\left(k \nabla p_{\varepsilon}^{\alpha}\right)\right|\left|\partial_{t} p_{\varepsilon}^{\alpha}\right| d x \\
& +C \frac{(M+\eta)^{3 / 2}}{m}\left(\int_{\Omega \cap B_{m}, M} \frac{\left.\left|T_{\eta}\left(\left|k^{\frac{1}{2}} \nabla p_{\varepsilon}^{\alpha}\right|^{2}\right)\right| d x\right)^{1 / 2}}{I_{\eta}}\right.
\end{aligned}
$$




$$
\begin{aligned}
& +C\left(\int_{\Omega}\left|T_{\eta}^{\prime}\left(\left|k^{\frac{1}{2}} \nabla p_{\varepsilon}^{\alpha}\right|^{2}\right)\right|\left|\underline{u}_{\varepsilon}\right||| \underline{u}_{\varepsilon}|-| \underline{u}_{\varepsilon}^{\alpha}||\left|\nabla p_{\varepsilon}^{\alpha}\right|^{2}\left|\nabla c_{\varepsilon}\right|^{2} d x\right)^{1 / 2} I_{\eta} \\
& +2\left|\int_{\Omega} \phi T_{\eta}^{\prime \prime}\left(\left|k^{\frac{1}{2}} \nabla p_{\varepsilon}^{\alpha}\right|^{2}\right) \frac{k}{\mu\left(c_{\varepsilon}\right)} \nabla p_{\varepsilon}^{\alpha} \cdot \nabla\left(\left|\nabla p_{\varepsilon}^{\alpha}\right|^{2}\right) \partial_{t} p_{\varepsilon}^{\alpha} d x\right| .
\end{aligned}
$$

Now we choose $m=2^{n}$ and $M=2^{n+1}, n \in \mathbb{N}$ and we let $\eta$ tend to 0 . Note that the function $T_{\eta}$ then converges to the function $T$ defined by

$$
T(x)= \begin{cases}x & \text { if } 2^{2 n} \leq x \leq 2^{2 n+2} \\ 0 & \text { elsewhere }\end{cases}
$$

We recall that $\lim _{\eta \rightarrow 0} I_{\eta}=0$. The last term of the right-hand side of Relation (3.13) also tends to zero with $\eta$ because $\phi T_{\eta}^{\prime \prime}\left(\left|k^{\frac{1}{2}} \nabla p_{\varepsilon}^{\alpha}\right|^{2}\right)\left(k / \mu\left(c_{\varepsilon}\right)\right)\left|\nabla p_{\varepsilon}^{\alpha}\right|^{2} \in L^{\infty}\left(\Omega_{T}\right)$, $\partial_{t} p_{\varepsilon}^{\alpha} \in L^{2}\left(\Omega_{T}\right)$ and $p_{\varepsilon}^{\alpha} \in L^{2}\left(0, T ; W^{2,2}(\Omega)\right)$. Then, letting $\eta \rightarrow 0$ in (3.14), we obtain

$$
\begin{aligned}
& \frac{d}{d t} \int_{\Omega} \frac{\phi}{\mu\left(c_{\varepsilon}\right)} T\left(\left|k^{\frac{1}{2}} \nabla p_{\varepsilon}^{\alpha}\right|^{2}\right) d x+\int_{\Omega} \phi_{-} T^{\prime}\left(\left|k^{\frac{1}{2}} \nabla p_{\varepsilon}^{\alpha}\right|^{2}\right) 2(1-\delta)\left|\partial_{t} p_{\varepsilon}^{\alpha}\right|^{2} d x \\
& \quad+\int_{\Omega}\left(\frac{\mu^{\prime \prime}}{\mu^{2}}\right)\left(c_{\varepsilon}\right) T\left(\left|k^{\frac{1}{2}} \nabla p_{\varepsilon}^{\alpha}\right|^{2}\right) \phi_{-}(1-\delta)\left(\varepsilon+\alpha_{T}\left|\underline{u}_{\varepsilon}\right|\right)\left|\nabla c_{\varepsilon}\right|^{2} d x \\
& \leq \frac{2^{n} C(t)}{\delta} \int_{\Omega} T\left(\left|k^{\frac{1}{2}} \nabla p_{\varepsilon}^{\alpha}\right|^{2}\right) d x+\frac{2^{n} C}{\delta}+C_{\alpha}(t),
\end{aligned}
$$

where $C(t)$ is uniformly bounded in $L^{1}(0, T)$. We have defined the quantity $C_{\alpha}$ by

$$
\begin{aligned}
C_{\alpha}(t)= & \frac{C}{\delta} \int_{\Omega}|| \underline{u}_{\varepsilon}|-| \underline{u}_{\varepsilon}^{\alpha}|| T\left(\left|k^{\frac{1}{2}} \nabla p_{\varepsilon}^{\alpha}\right|^{2}\right) d x \\
& +C \int_{\Omega}\left|\frac{1}{\mu\left(c_{\varepsilon}^{\alpha}\right)}-\frac{1}{\mu\left(c_{\varepsilon}\right)}\right| T^{\prime}\left(\left|k^{\frac{1}{2}} \nabla p_{\varepsilon}^{\alpha}\right|^{2}\right)\left(\varepsilon+\alpha_{L}\left|\underline{u}_{\varepsilon}\right|\right)\left|\nabla p_{\varepsilon}^{\alpha}\right|^{2}\left|\nabla c_{\varepsilon}\right|^{2} d x \\
& +C \int_{\Omega}\left|\mu^{\prime}\left(c_{\varepsilon}^{\alpha}\right)-\mu^{\prime}\left(c_{\varepsilon}\right)\right| T^{\prime}\left(\left|k^{\frac{1}{2}} \nabla p_{\varepsilon}^{\alpha}\right|^{2}\right)\left|k^{\frac{1}{2}} \nabla p_{\varepsilon}^{\alpha}\right|^{2}\left(\varepsilon+\alpha_{L}\left|\underline{u}_{\varepsilon}\right|\right)\left|\nabla c_{\varepsilon}\right|^{2} d x \\
& +\frac{C}{\delta} \int_{\Omega} T^{\prime}\left(\left|k^{\frac{1}{2}} \nabla p_{\varepsilon}^{\alpha}\right|^{2}\right)\left|\underline{u}_{\varepsilon}\right||| \underline{u}_{\varepsilon}|-| \underline{u}_{\varepsilon}^{\alpha}||\left|\nabla p_{\varepsilon}^{\alpha}\right|^{2}\left(\left|\nabla c_{\varepsilon}\right|^{2}+\left|\nabla c_{\varepsilon}^{\alpha}\right|^{2}\right) d x \\
& +C \int_{\Omega} T^{\prime}\left(\left|k^{\frac{1}{2}} \nabla p_{\varepsilon}^{\alpha}\right|^{2}\right)\left|-\frac{\mu^{\prime}\left(c_{\varepsilon}^{\alpha}\right)}{\left.\mu^{(} c_{\varepsilon}^{\alpha}\right)} \nabla c_{\varepsilon}^{\alpha}+\frac{\mu^{\prime}\left(c_{\varepsilon}\right)}{\left.\mu^{(} c_{\varepsilon}\right)} \nabla c_{\varepsilon}\right|\left|\nabla p_{\varepsilon}^{\alpha}\right|\left|\partial_{t} p_{\varepsilon}^{\alpha}\right| d x \\
& +\int_{\Omega} \phi\left|T_{\eta}^{\prime}\left(\left|k^{\frac{1}{2}} \nabla p_{\varepsilon}^{\alpha}\right|^{2}\right)\right|\left|\frac{1}{\mu\left(c_{\varepsilon}^{\alpha}\right)}-\frac{1}{\mu\left(c_{\varepsilon}\right)}\right|\left|\operatorname{div}\left(k \nabla p_{\varepsilon}^{\alpha}\right)\right|\left|\partial_{t} p_{\varepsilon}^{\alpha}\right| d x .
\end{aligned}
$$

In view of the results of Lemma 3 , for any fixed $\varepsilon>0$, all the integrals in (3.16) are of the form $\int_{\Omega}\left(f_{\varepsilon}^{\alpha}-f_{\varepsilon}\right) g_{\varepsilon}^{\alpha} d x$ where $f_{\varepsilon}^{\alpha}$ converges to $f_{\varepsilon}$ almost everywhere in $\Omega_{T}$ and $g_{\varepsilon}^{\alpha}$ is uniformly bounded with respect to $\alpha$ in $L^{1}\left(\Omega_{T}\right)$. We conclude that for any $t \in(0, T)$ and any fixed $\varepsilon>0$,

$$
\lim _{\alpha \rightarrow 0} \int_{0}^{t} C_{\alpha}(s) d s=0 .
$$


Let us turn back to Relation (3.15). We recall that by (1.7), $\mu^{\prime \prime}(c) / \mu^{2}(c) \geq \mu_{-}>0$. One can choose $\delta>0$ such that $1-2 \delta>\delta_{o}>0$. Using the Gronwall lemma on Relation (3.15), we then get

$$
\begin{aligned}
\int_{\Omega} \frac{\phi}{\mu\left(c_{\varepsilon}\right)} T\left(\left|k^{\frac{1}{2}} \nabla p_{\varepsilon}^{\alpha}\right|^{2}\right) d x \leq & e^{2^{n} t} \int_{\Omega} \frac{\phi}{\mu\left(c_{\varepsilon}\right)} T\left(\left|k^{\frac{1}{2}} \nabla p_{\text {init }}^{\alpha}\right|^{2}\right) d x \\
& +C+\int_{0}^{t} C_{\alpha}(s) e^{-2^{n}(s-t)} d s .
\end{aligned}
$$

Thus, using Assumption (1.11), we conclude that

$$
\int_{\Omega_{t}} T^{\prime}\left(\left|k^{\frac{1}{2}} \nabla p_{\varepsilon}^{\alpha}\right|^{2}\right)\left|\partial_{t} p_{\varepsilon}^{\alpha}\right|^{2} d x d s \leq 2^{n} C+\int_{0}^{t}\left|C_{\alpha}(s)\right| e^{-2^{n}(s-t)} d s .
$$

Besides, letting $\eta$ to 0 in (3.9) and using the latter result, we obtain

$$
\begin{aligned}
\sum_{i, j=1}^{N} \int_{\Omega_{t}} T^{\prime}\left(\left|k^{\frac{1}{2}} \nabla p_{\varepsilon}^{\alpha}\right|^{2}\right)\left|\partial_{i} \underline{u}_{\varepsilon, j}^{\alpha}\right|^{2} d x d s \leq & \int_{\Omega_{t}} T^{\prime}\left(\left|k^{\frac{1}{2}} \nabla p_{\varepsilon}^{\alpha}\right|^{2}\right)\left(\operatorname{div}\left(\underline{u}_{\varepsilon}^{\alpha}\right)^{2}\right. \\
& \left.+\left|\operatorname{curl}\left(\underline{u}_{\varepsilon}^{\alpha}\right)\right|^{2}\right) d x d s \\
\leq & C \int_{\Omega_{t}} T^{\prime}\left(\left|k^{\frac{1}{2}} \nabla p_{\varepsilon}^{\alpha}\right|^{2}\right)\left(\left|\partial_{t} p_{\varepsilon}^{\alpha}\right|^{2}\right. \\
& \left.+\left(\left|\nabla c_{\varepsilon}^{\alpha}\right|+1\right)^{2}\left|\nabla p_{\varepsilon}^{\alpha}\right|^{2}\right) d x d s \\
\leq & 2^{n} C+C \int_{0}^{t}\left|C_{\alpha}(s)\right| e^{-2^{n}(s-t)} d s \\
& +2^{n} C \int_{\Omega_{t}}\left|\underline{u}_{\varepsilon}^{\alpha}\right|\left|\nabla c_{\varepsilon}^{\alpha}\right|^{2} d x d s+C \\
\leq & 2^{n} C+C \int_{0}^{t}\left|C_{\alpha}(s)\right| e^{-2^{n}(s-t)} d s .
\end{aligned}
$$

Now we let $\alpha$ tend to 0 in the latter relation. In view of the result (3.17) and of the strong convergences stated in Lemma 3 (iii), we conclude that for any $n \in \mathbb{N}$ and $1 \leq i, j \leq N$

$$
\left\|\partial_{i} \underline{u}_{\varepsilon, j}\right\|_{L^{2}\left(B_{n}\right)}^{2} \leq 2^{n} C,
$$

where the set $B_{n}$ is defined by $B_{n}=\left\{(x, t) \in \Omega_{T} ; 2^{n} \leq k^{\frac{1}{2}}\left|\nabla p_{\varepsilon}(x, t)\right| \leq 2^{n+1}\right\}$.

We finish with some words about the estimate in $B$ announced in the lemma. We do not detail its proof because it is completely similar to the one carried for the estimate in $B_{n}$. One simply has to follow the previous lines, choosing $m=0$ and $M=1$. Furthermore, the proof in this case is more simple because one does not have to introduce the technical artifice of dividing by $m$ (in (3.8) for instance). Indeed, we have here $M^{2}=M=1$. This ends the proof of the lemma.

The second step of our renormalization method is the following lemma.

Lemma 5. The sequence $\left(\underline{u}_{\varepsilon}\right)$ is uniformly bounded in $L^{\theta}\left(0, T ; W^{1, \theta}(\Omega)\right)$ for any real $\theta$ such that $1 \leq \theta<(6 N+12) /(5 N+8)$. 
Proof. Let $\theta$ be a real number, $1 \leq \theta<2$. Using the Hölder inequality, we write

$$
\int_{B_{n}}\left|\partial_{i} \underline{u}_{\varepsilon, j}\right|^{\theta} d x d t \leq\left(\int_{B_{n}}\left|\partial_{i} \underline{u}_{\varepsilon, j}\right|^{2} d x d t\right)^{\theta / 2}\left|B_{n}\right|^{1-\theta / 2},
$$

where $\left|B_{n}\right|$ denotes the measure of $B_{n}$. We note that on $B_{n}$, we have $\left|\underline{u}_{\varepsilon}\right| \geq$ $C 2^{n}$ where the constant $C$ depends only on variations of the functions $k$ and $\mu$. Therefore,

$$
\left|B_{n}\right| \leq \frac{C}{2^{n}} \int_{B_{n}}\left|\underline{u}_{\varepsilon}(x, t)\right| d x d t .
$$

By the Hölder inequality, we also have

$$
\int_{B_{n}}\left|\underline{u}_{\varepsilon}(x, t)\right| d x d t \leq\left(\int_{B_{n}}\left|\underline{u}_{\varepsilon}(x, t)\right|^{s} d x d t\right)^{1 / s}\left|B_{n}\right|^{1 / s^{\prime}},
$$

for any $s, s^{\prime}>1$ with $1 / s+1 / s^{\prime}=1$. This produces

$$
\left|B_{n}\right| \leq \frac{C}{2^{n s}} \int_{B_{n}}\left|\underline{u}_{\varepsilon}(x, t)\right|^{s} d x d t .
$$

Thus, in view of inequality (3.18),

$$
\int_{B_{n}}\left|\partial_{i} \underline{u}_{\varepsilon, j}(x, t)\right|^{\theta} d x d t \leq \frac{C}{2^{n(s(1-\theta / 2)-\theta / 2)}}\left(\int_{B_{n}}\left|\underline{u}_{\varepsilon}(x, t)\right|^{s} d x d t\right)^{1-\theta / 2}
$$

and then

$$
\begin{aligned}
\sum_{n \geq 0} \int_{B_{n}}\left|\partial_{i} \underline{u}_{\varepsilon, j}(x, t)\right|^{\theta} & d x d t \\
& \leq \sum_{n \geq 0} \frac{C}{2^{n(s(1-\theta / 2)-\theta / 2)}}\left(\int_{B_{n}}\left|\underline{u}_{\varepsilon}(x, t)\right|^{s} d x d t\right)^{1-\theta / 2} .
\end{aligned}
$$

We now choose $s>\theta /(2-\theta)$. Using the discrete Hölder inequality, the right-hand side of inequality (3.19) is majorized as

$$
\begin{aligned}
& \sum_{n \geq 0} \frac{1}{2^{n(s(1-\theta / 2)-\theta / 2)}}\left(\int_{B_{n}}\left|\underline{u}_{\varepsilon}\right|^{s} d x d t\right)^{1-\theta / 2} \\
& \quad \leq\left(\sum_{n \geq 0} \frac{1}{2^{n r(s(1-\theta / 2)-\theta / 2)}}\right)^{1 / r}\left(\sum_{n \geq 0}\left(\int_{B_{n}}\left|\underline{u}_{\varepsilon}\right|^{s} d x d t\right)^{(1-\theta / 2) r^{\prime}}\right)^{1 / r^{\prime}}
\end{aligned}
$$


with $1 / r+1 / r^{\prime}=1$. Choosing $r^{\prime}=2 /(2-\theta)$, we infer from $(3.19)$

$$
\begin{aligned}
\sum_{n \geq 0} \int_{B_{n}}\left|\partial_{i} \underline{u}_{\varepsilon, j}\right|^{\theta} d x d t & \leq C\left(\sum_{n \geq 0} \int_{B_{n}}\left|\underline{u}_{\varepsilon}\right|^{s} d x d t\right)^{1-\theta / 2} \\
& \leq C\left(\int_{\Omega_{T}}\left|\underline{u}_{\varepsilon}\right|^{s} d x d t\right)^{1-\theta / 2} \quad \forall 1 \leq i, j \leq N .
\end{aligned}
$$

Now we recall that $\int_{B}\left|\partial_{i} \underline{u}_{\varepsilon, j}\right|^{2} d x d t \leq C$. So, with (3.20), we have established the estimate

$$
\int_{\Omega_{T}}\left|\partial_{i} \underline{u}_{\varepsilon, j}\right|^{\theta} d x d t \leq C\left(1+\left(\int_{\Omega_{T}}\left|\underline{u}_{\varepsilon}\right|^{s} d x d t\right)^{1-\theta / 2}\right) \quad \forall 1 \leq i, j \leq N,
$$

for any $s>\theta /(2-\theta)$. We now write the following Gagliardo-Nirenberg inequality

$$
\left\|\underline{u}_{\varepsilon}\right\|_{L^{s}(\Omega)} \leq\left\|\underline{u}_{\varepsilon}\right\|_{W^{1, \theta}(\Omega)}^{1 / 2}\left\|p_{\varepsilon}\right\|_{L^{2(N+2) / N}(\Omega)}^{1 / 2},
$$

where $s$ is such that $\frac{1}{s}=\frac{1}{2}\left(\frac{N}{2(N+2)}+\frac{1}{\theta}\right)$, that is $s=4 \theta(N+2) /(N \theta+2(N+2))$. The condition $s>\theta /(2-\theta)$ is fulfilled provided that $\theta<(6 N+12) /(5 N+8)$. Integrating from 0 to $T$ and using the Hölder inequality, we deduce that

$$
\begin{aligned}
\int_{\Omega_{T}}\left|\underline{u}_{\varepsilon}\right|^{s} d x d t & \leq \int_{0}^{T}\left(\int_{\Omega}\left|\partial_{i} \underline{u}_{\varepsilon, j}\right|^{\theta} d x\right)^{\frac{2(N+2)}{N \theta+2(N+2)}}\left(\int_{\Omega}\left|p_{\varepsilon}\right|^{\frac{2(N+2)}{N}} d x\right)^{\frac{N \theta}{N \theta+2(N+2)}} d t \\
\leq & \left(\int_{0}^{T} \int_{\Omega}\left|\partial_{i} \underline{u}_{\varepsilon, j}\right|^{\theta} d x d t\right)^{\frac{2(N+2)}{N \theta+2(N+2)}} \\
& \times\left(\int_{0}^{T} \int_{\Omega}\left|p_{\varepsilon}\right|^{\frac{2(N+2)}{N}} d x d t\right)^{\frac{N \theta}{N \theta+2(N+2)}} .
\end{aligned}
$$

We recall that the function $p_{\varepsilon}$ is uniformly bounded in the space $L^{2}\left(0, T ; H^{1}(\Omega)\right) \subset$ $L^{2}\left(0, T ; L^{2 N /(N-2)}(\Omega)\right)$ and in $L^{\infty}\left(0, T ; L^{2}(\Omega)\right)$. By interpolation, $p_{\varepsilon}$ is thus also uniformly bounded in $L^{2(N+2) / N}\left(\Omega_{T}\right)$. Thus, using the latter relation in (3.20), we obtain

$$
\int_{\Omega_{T}}\left|\partial_{i} \underline{u}_{\varepsilon, j}\right|^{\theta} d x d t \leq C+C\left(\int_{\Omega_{T}}\left|\partial_{i} \underline{u}_{\varepsilon, j}\right|^{\theta} d x d t\right)^{\frac{2(N+2)}{N \theta+2(N+2)}} .
$$

Since $2(N+2) /(N \theta+2(N+2))<1$, the latter relation gives the result of the lemma.

\section{Convergence results as $\varepsilon \rightarrow 0$ and proof of Theorem 2}

The results of the previous sections and classical Sobolev embedding allow to assert the existence of $p \in \mathrm{E}^{\infty}\left(0, T ; L^{2}(\Omega)\right) \cap L^{2}\left(0, T ; H^{1}(\Omega)\right) \cap W^{1, \theta}\left(0, T ; L^{\theta}(\Omega)\right) \cap$ 
$L^{\theta}\left(0, T ; W^{2,4 / 3}(\Omega)\right), \underline{u} \in L^{2}\left(\Omega_{T}\right) \cap L^{\theta}\left(0, T ; W^{1, \theta}(\Omega)\right)$ and $c \in L^{\infty}\left(\Omega_{T}\right)$ such that, for extracted subsequences not relabeled for convenience,

$$
\begin{aligned}
p_{\varepsilon} & \rightarrow p \quad \text { a.e. in } \Omega_{T}, \\
\partial_{t} p_{\varepsilon} & \rightarrow \partial_{t} p \text { weakly in } L^{\theta}\left(\Omega_{T}\right), \\
\nabla p_{\varepsilon} & \rightarrow \nabla p \quad \text { weakly in }\left(L^{2}\left(\Omega_{T}\right)\right)^{N} \cap\left(L^{\theta}\left(0, T ; W^{1,4 / 3}(\Omega)\right)\right)^{N}, \\
c_{\varepsilon} & \rightarrow c * \text { - weakly in } L^{\infty}\left(\Omega_{T}\right),
\end{aligned}
$$

and these functions satisfy the following limit equations in $\Omega_{T}$

$$
\begin{aligned}
& \phi \partial_{t} p+\operatorname{div}(\underline{u})=0, \quad \underline{u}=-k \overline{\frac{1}{\mu\left(c_{\varepsilon}\right)} \nabla p_{\varepsilon}}, \\
& \phi \partial_{t} c+\overline{u_{\varepsilon} \cdot \nabla c_{\varepsilon}}-\operatorname{div}\left(\overline{D_{p}\left(\underline{u}_{\varepsilon}\right) \nabla c_{\varepsilon}}\right)=0,
\end{aligned}
$$

where $\overline{f_{\varepsilon}}$ denotes the weak ad hoc limit of $f_{\varepsilon}$.

Our first step is to explicit $\overline{u_{\varepsilon} c_{\varepsilon}}$.

Lemma 6. Up to extracted subsequences, we have

$$
\underline{u}_{\varepsilon} c_{\varepsilon} \rightarrow \underline{u} c \text { weakly in }\left(L^{2}\left(\Omega_{T}\right)\right)^{N} .
$$

Proof. Multiplying Eq. (1.4) by a function $\psi \in L^{4}\left(0, T ; W^{1,4}(\Omega)\right)$, one easily checks that the function $\phi \partial_{t} c_{\varepsilon}$ is uniformly bounded in $L^{4 / 3}\left(0, T ;\left(W^{1,4}(\Omega)\right)^{\prime}\right)$. By Lemma $2, \phi c_{\varepsilon} \rightarrow \phi c$ weakly in $L^{q}\left(0, T ; L^{2}(\Omega)\right)$ for any $q \geq 1$. By Lemmas 1 and $5, \underline{u}_{\varepsilon} \rightarrow \underline{u}$ weakly in $\left(L^{\theta}\left(0, T ; W^{1, \theta}(\Omega)\right)\right)^{N} \cap\left(L^{2}\left(\Omega_{T}\right)\right)^{N}$. Since we have the following embedding

$$
W^{1, \theta}(\Omega) \subset L^{2}(\Omega)=\left(L^{2}(\Omega)\right)^{\prime} \subset\left(W^{1, \theta}(\Omega)\right)^{\prime} \subset\left(W^{1,4}(\Omega)\right)^{\prime},
$$

the embedding $W^{1, \theta}(\Omega) \subset L^{2}(\Omega)$ being compact, we can use a Aubin's type compactness argument (see [9] Lemma 6 ) to assert that

$$
\underline{u}_{\varepsilon} \phi c_{\varepsilon} \rightarrow \underline{u} \phi c \text { in }\left(\mathcal{D}^{\prime}\left(\Omega_{T}\right)\right)^{N} .
$$

The result of the lemma follows from the property $\phi(x) \geq \phi_{-}>0$ in $\Omega$.

Now, we are able to give an explicit value for the limit velocity.

Lemma 7. The limit velocity is defined by the following Darcy law.

$$
\underline{u}=-\frac{k}{\mu(c)} \nabla p .
$$


Proof. Let us write the equations satisfied by the functions $\mu\left(c_{\varepsilon}\right)$ and $1 / \mu\left(c_{\varepsilon}\right)$ in $\Omega_{T}$.

$$
\begin{aligned}
& \phi \partial_{t} \mu\left(c_{\varepsilon}\right)+\underline{u}_{\varepsilon} \cdot \nabla \mu\left(c_{\varepsilon}\right)-\operatorname{div}\left(\phi\left(\varepsilon I d+D_{p}\left(\underline{u}_{\varepsilon}\right)\right) \nabla \mu\left(c_{\varepsilon}\right)\right) \\
&=-\mu^{\prime \prime}\left(c_{\varepsilon}\right) \phi\left(\varepsilon I d+D_{p}\left(\underline{u}_{\varepsilon}\right)\right) \nabla c_{\varepsilon} \cdot \nabla c_{\varepsilon} \\
& \phi \partial_{t} \frac{1}{\mu\left(c_{\varepsilon}\right)}+\underline{u}_{\varepsilon} \cdot \nabla \frac{1}{\mu\left(c_{\varepsilon}\right)}-\operatorname{div}\left(\phi\left(\varepsilon I d+D_{p}\left(\underline{u}_{\varepsilon}\right)\right) \nabla \frac{1}{\mu\left(c_{\varepsilon}\right)}\right) \\
&=\frac{\mu^{\prime \prime}\left(c_{\varepsilon}\right) \mu\left(c_{\varepsilon}\right)-2 \mu^{\prime}\left(c_{\varepsilon}\right)^{2}}{\mu\left(c_{\varepsilon}\right)^{3}} \phi\left(\varepsilon I d+D_{p}\left(\underline{u}_{\varepsilon}\right)\right) \nabla c_{\varepsilon} \cdot \nabla c_{\varepsilon} .
\end{aligned}
$$

We note that the structure of these equations is completely similar to the one of (1.4), with right-hand sides in $L^{1}\left(\Omega_{T}\right)$. One easily checks that $\phi \partial_{t} \mu\left(c_{\varepsilon}\right)$ and $\phi \partial_{t}\left(1 / \mu\left(c_{\varepsilon}\right)\right)$ are uniformly bounded in $L^{1}\left(0, T ;\left(W^{1,4}(\Omega)\right)^{\prime}\right)$. We then use the same tools as in the proof of the previous lemma for the sequences $\left(\phi \mu\left(c_{\varepsilon}\right)\right)$ (respectively $\left.\phi / \mu\left(c_{\varepsilon}\right)\right)$ and $\underline{u}_{\varepsilon}$ (respectively $\nabla p_{\varepsilon}$ bounded in $L^{\theta}\left(0, T ; W^{1,4 / 3}(\Omega)\right)$ with the compact embedding $\left.W^{1,4 / 3}(\Omega) \subset L^{2}(\Omega)\right)$. We conclude that

$$
\underline{u}_{\varepsilon} \mu\left(c_{\varepsilon}\right) \rightarrow \underline{u} \overline{\mu\left(c_{\varepsilon}\right)}, \quad \nabla p_{\varepsilon} \frac{1}{\mu\left(c_{\varepsilon}\right)} \rightarrow \nabla p \overline{\frac{1}{\mu\left(c_{\varepsilon}\right)}} \quad \text { in } \quad\left(L^{2}\left(\Omega_{T}\right)\right)^{N} .
$$

Since we also know the weak convergences $\underline{u}_{\varepsilon} \mu\left(c_{\varepsilon}\right)=-k \nabla p_{\varepsilon} \rightarrow-k \nabla p$ and $\nabla p_{\varepsilon}\left(1 / \mu\left(c_{\varepsilon}\right)\right)=-k^{-1} \underline{u}_{\varepsilon} \rightarrow-k^{-1} \underline{u}$ in $\left(L^{2}\left(\Omega_{T}\right)\right)^{N}$, we conclude that on the one hand

$$
\underline{u}=-\frac{k}{\overline{\mu\left(c_{\varepsilon}\right)}} \nabla p=-k \overline{\frac{1}{\mu\left(c_{\varepsilon}\right)}} \nabla p .
$$

On the other hand, since the functions $\mu$ and $1 / \mu$ are convex, we can assert that $\left(\overline{\frac{1}{\mu\left(c_{\varepsilon}\right)}}\right)^{-1} \leq \mu(c) \leq \overline{\mu\left(c_{\varepsilon}\right)}$. Relation (4.1) thus implies that

$$
\underline{u}=-\frac{k}{\overline{\mu\left(c_{\varepsilon}\right)}} \nabla p=-k \overline{\frac{1}{\mu\left(c_{\varepsilon}\right)}} \nabla p=\frac{-k}{\mu(c)} \nabla p .
$$

Lemma 7 is proven.

The result of the previous lemma let us claim that the limit pressure is solution of (1.12). We now prove the following compactness results.

Lemma 8. We claim that, up to extracted subsequences,

$$
\begin{gathered}
\nabla p_{\varepsilon} \rightarrow \nabla p \quad \text { strongly in }\left(L^{2}\left(\Omega_{T}\right)\right)^{N} \quad \text { and a.e. in } \Omega_{T}, \\
\underline{u}_{\varepsilon} \rightarrow \underline{u} \quad \text { strongly in } \quad\left(L^{2}\left(\Omega_{T}\right)\right)^{N} \text { and a.e. in } \Omega_{T},
\end{gathered}
$$


Proof. We multiply Eq. (1.3) by $p_{\varepsilon}$ and Eq. (1.12) by $p$. Integrating by parts over $\Omega_{t}=\Omega \times(0, t)$ for any $t \in(0, T)$, we obtain

$$
\begin{gathered}
\frac{1}{2} \int_{\Omega} \phi\left|p_{\varepsilon}(\cdot, t)\right|^{2} d x-\frac{1}{2} \int_{\Omega} \phi\left|p_{\text {init }}(\cdot)\right|^{2} d x+\int_{\Omega_{t}} \frac{k}{\mu\left(c_{\varepsilon}\right)} \nabla p_{\varepsilon} \cdot \nabla p_{\varepsilon} d x d s=0, \\
\frac{1}{2} \int_{\Omega} \phi|p(\cdot, t)|^{2} d x-\frac{1}{2} \int_{\Omega} \phi\left|p_{\text {init }}(\cdot)\right|^{2} d x+\int_{\Omega_{t}} \frac{k}{\mu(c)} \nabla p \cdot \nabla p d x d s=0 .
\end{gathered}
$$

Thus, using the a.e. convergence of $p_{\varepsilon}$ to $p$ in $\Omega_{T}$, we conclude that

$$
\lim _{\varepsilon \rightarrow 0} \int_{\Omega_{t}} \frac{k}{\mu\left(c_{\varepsilon}\right)} \nabla p_{\varepsilon} \cdot \nabla p_{\varepsilon} d x d s=\int_{\Omega_{t}} \frac{k}{\mu(c)} \nabla p \cdot \nabla p d x d s .
$$

Using the latter relation and the two last expressions of $\underline{u}$ given in (4.2), we now compute

$$
\begin{aligned}
\lim _{\varepsilon \rightarrow 0} \int_{\Omega_{t}} \frac{k}{\mu\left(c_{\varepsilon}\right)}\left|\nabla p_{\varepsilon}-\nabla p\right|^{2} d x d s= & \lim _{\varepsilon \rightarrow 0}\left(\int_{\Omega_{t}} \frac{k}{\mu\left(c_{\varepsilon}\right)}\left|\nabla p_{\varepsilon}\right|^{2} d x d s\right. \\
& +\int_{\Omega_{t}} \frac{k}{\mu\left(c_{\varepsilon}\right)}|\nabla p|^{2} d x d s-\int_{\Omega_{t}} \underline{u}_{\varepsilon} \cdot \nabla p d x d s \\
& \left.-\int_{\Omega_{t}} \underline{u}_{\varepsilon} \cdot \nabla p d x d s\right) \\
= & \int_{\Omega_{t}} \frac{k}{\mu(c)}|\nabla p|^{2} d x d s+\int_{\Omega_{t}} k \frac{1}{\mu\left(c_{\varepsilon}\right)}|\nabla p|^{2} d x d s \\
& -\int_{\Omega_{t}} k \frac{1}{\mu\left(c_{\varepsilon}\right)}|\nabla p|^{2} d x d s \\
& -\int_{\Omega_{t}} \frac{k}{\mu(c)}|\nabla p|^{2} d x d s=0 .
\end{aligned}
$$

Since $k / \mu\left(c_{\varepsilon}\right) \geq k_{-} / \mu_{+}>0$ almost everywhere in $\Omega_{T}$, the latter relation proves the strong convergence of $\nabla p_{\varepsilon}$ to $\nabla p$ in $\left(L^{2}\left(\Omega_{T}\right)\right)^{N}$.

Let us consider the behavior of $\left(\underline{u}_{\varepsilon}\right)$. Since we now know that $\nabla p_{\varepsilon}$ strongly converges to $\nabla p$ in $\left(L^{2}\left(\Omega_{T}\right)\right)^{N}$, we can assert that the weak limit in $L^{1}\left(\Omega_{T}\right)$ of $\mu\left(c_{\varepsilon}\right) \underline{u}_{\varepsilon} \cdot \underline{u}_{\varepsilon}=-k \nabla p_{\varepsilon} \cdot \underline{u}_{\varepsilon}$ is $-k \nabla p \cdot \underline{u}=\mu(c) \underline{u} \cdot \underline{u}=\overline{\mu\left(c_{\varepsilon}\right)} \underline{u} \cdot \underline{u}$ (see (4.2)). We thus compute

$$
\begin{aligned}
\lim _{\varepsilon \rightarrow 0} \int_{\Omega_{T}} \mu\left(c_{\varepsilon}\right)\left|\underline{u}_{\varepsilon}-\underline{u}\right|^{2} d x d t= & \lim _{\varepsilon \rightarrow 0}\left(\int_{\Omega_{T}} \mu\left(c_{\varepsilon}\right) \underline{u}_{\varepsilon} \cdot \underline{u}_{\varepsilon} d x d t+\int_{\Omega_{T}} \mu\left(c_{\varepsilon}\right) \underline{u} \cdot \underline{u} d x d t\right. \\
& \left.-\int_{\Omega_{T}} \mu\left(c_{\varepsilon}\right) \underline{u} \varepsilon \cdot \underline{u} d x d t-\int_{\Omega_{T}} \mu\left(c_{\varepsilon}\right) \underline{u}_{\varepsilon} \cdot \underline{u} d x d t\right) \\
= & \int_{\Omega_{T}} \mu(c) \underline{u} \cdot \underline{u} d x d t+\int_{\Omega_{T}} \overline{\mu\left(c_{\varepsilon}\right)} \underline{u} \cdot \underline{u} d x d t \\
& -\int_{\Omega_{T}} \overline{\mu\left(c_{\varepsilon}\right)} \underline{u} \cdot \underline{u} d x d t-\int_{\Omega_{T}} \mu(c) \underline{u} \cdot \underline{u} d x d t=0 .
\end{aligned}
$$


Since $\mu\left(c_{\varepsilon}\right) \geq \mu_{-}>0$ almost everywhere in $\Omega_{T}$, the latter relation proves the strong convergence of $\underline{u}_{\varepsilon}$ to $\underline{u}$ in $\left(L^{2}\left(\Omega_{T}\right)\right)^{N}$.

We now aim to explicit the limit convection term $\overline{u_{\varepsilon} \cdot \nabla c_{\varepsilon}}$.

Lemma 9. The following convergence hold true.

$$
\underline{u}_{\varepsilon} \cdot \nabla c_{\varepsilon} \rightarrow \underline{u} \cdot \nabla c \quad \text { weakly in } L^{4 / 3}\left(\Omega_{T}\right) .
$$

Proof. We begin by dividing $\Omega_{T}$ in two sets:

$$
\Omega_{T}=D \cup D^{c}, \quad \text { with } \quad D=\left\{(x, t) \in \Omega_{T} ;|\underline{u}(x, t)| \neq 0\right\} .
$$

Obviously we have

$$
\lim _{\varepsilon \rightarrow 0} \underline{u}_{\varepsilon} \cdot \nabla c_{\varepsilon}=0=\underline{u} \cdot \nabla c \quad \text { in } \quad L^{4 / 3}\left(D^{c}\right)-\text { weak } .
$$

We now consider the convergence problem in $D$. Writing $\underline{u}_{\varepsilon} \cdot \nabla c_{\varepsilon}=\operatorname{div}\left(\underline{u}_{\varepsilon} c_{\varepsilon}\right)+$ $c_{\varepsilon} \partial_{t} p_{\varepsilon}$ and bearing in mind Lemma 6 , it reminds to explicit the limit of $c_{\varepsilon} \partial_{t} p_{\varepsilon}$ in $L^{\theta}(D)$. Let $f$ be the function defined in $\mathbb{R}_{+}$by $f(x)=(x+1)^{\theta / 2(\theta-1)}$. Note that the functions $f$ and $1 / f$ are convex since $1 \leq \theta<2$. We also define a sequence $v_{\varepsilon}$ by

$$
v_{\varepsilon}=f\left(c_{\varepsilon}\right)\left|\underline{u}_{\varepsilon}\right| .
$$

We then exactly follow the lines of the proof of Lemma 7 (except that we now know that $\underline{u}_{\varepsilon}$ strongly converges to $\underline{u}$ ) to conclude that

$$
|\underline{u}|=\frac{1}{f(c)} v=\frac{1}{\overline{f\left(c_{\varepsilon}\right)}} v=\overline{\frac{1}{f\left(c_{\varepsilon}\right)}} v .
$$

We then compute

$$
\begin{aligned}
\lim _{\varepsilon \rightarrow 0} \int_{\Omega_{T}} \frac{1}{f\left(c_{\varepsilon}\right)}\left|v_{\varepsilon}-v\right|^{2} d x d t= & \lim _{\varepsilon \rightarrow 0}\left(\int_{\Omega_{T}}\left|\underline{u}_{\varepsilon}\right| v_{\varepsilon} d x d t+\int_{\Omega_{T}} \frac{1}{f\left(c_{\varepsilon}\right)} v^{2} d x d t\right. \\
& \left.-2 \int_{\Omega_{T}}\left|\underline{u}_{\varepsilon}\right| v d x d t\right)=0 .
\end{aligned}
$$

We infer from the latter relation

$$
v_{\varepsilon}=f\left(c_{\varepsilon}\right)\left|\underline{u}_{\varepsilon}\right| \rightarrow v=f(c)|\underline{u}| \quad \text { in } \quad L^{2}\left(\Omega_{T}\right) \text { and a.e. in } \Omega_{T} .
$$

We then can pass to the limit in the product $v_{\varepsilon}^{2(\theta-1) / \theta} \partial_{t} p_{\varepsilon}$ which is uniformly bounded in $L^{1}\left(\Omega_{T}\right)$. We get

$$
v_{\varepsilon}^{2(\theta-1) / \theta} \partial_{t} p_{\varepsilon}=\left(c_{\varepsilon}+1\right)\left|\underline{u}_{\varepsilon}\right|^{2(\theta-1) / \theta} \partial_{t} p_{\varepsilon} \rightarrow v^{2(\theta-1) / \theta} \partial_{t} p=(c+1)|\underline{u}|^{2(\theta-1) / \theta} \partial_{t} p .
$$

Since $\left|u_{\varepsilon}\right|$ converges almost everywhere in $\Omega_{T}$ to $|\underline{u}|$, the latter result implies that

$$
c_{\varepsilon} \partial_{t} p_{\varepsilon} \rightarrow c \partial_{t} p \quad \text { in } \quad L^{\theta}(D)
$$

and then

$$
\underline{u}_{\varepsilon} \cdot \nabla c_{\varepsilon}=\operatorname{div}\left(\underline{u}_{\varepsilon} c_{\varepsilon}\right)+c_{\varepsilon} \partial_{t} p_{\varepsilon} \rightarrow \underline{u} \cdot \nabla c=\operatorname{div}(\underline{u} c)+c \partial_{t} p \quad \text { in } \quad L^{4 / 3}(D) .
$$

Lemma 9 is proven. 
Finally we note that the same arguments as in the latter proof lead to

$$
\underline{u}_{\varepsilon, j} \partial_{i} c_{\varepsilon} \rightarrow \underline{u}_{j} \partial_{i} c \quad \text { in } \quad L^{4 / 3}\left(\Omega_{T}\right), \quad \forall 1 \leq i, j \leq N .
$$

Using besides the a.e. convergence of $\underline{u}_{\varepsilon}$ to $\underline{u}$, we conclude that

$$
D_{p}\left(\underline{u}_{\varepsilon}^{\alpha}\right) \nabla c_{\varepsilon} \rightarrow D_{p}(\underline{u}) \nabla c \quad \text { in } \quad\left(L^{4 / 3}\left(\Omega_{T}\right)\right)^{N} .
$$

This ends the proof of Theorem 2 .

\section{References}

[1] Y. Amirat, K. Hamdache, and A. Ziani. Mathematical analysis for compressible miscible displacement models in porous media. Math. Models Methods Appl. Sci., 6(6):729-747, 1996

[2] Y. Amirat and M. Moussaoui. Analysis of a one-dimensional model for compressible miscible displacement in porous media. SIAM J. Math. Anal., 26:659-674, 1995.

[3] Y. Amirat and A. Ziani. Asymptotic behavior of the solutions of an elliptic-parabolic system arising in flow in porous media. Z. Anal. Anwendungen, 23(2):335-351, 2004.

[4] J. Bear. Dynamics of Fluids in Porous Media, American Elsevier, 1972.

[5] C. Choquet. Existence result for a radionuclide transport model with an unbounded viscosity. J. Math. Fluid Mech., 6(4):365-388, 2004.

[6] C. Choquet. On a nonlinear parabolic system modelling miscible compressible displacement in porous media. Nonlinear Anal., 61(1-2):237-260, 2005.

[7] G. de Marsily. Hydrogéologie quantitative, Masson, 1981.

[8] F. J. Fayers. An approximate model with physically interpretable parameters for representing miscible viscous fingering. SPERE, 3:551-558, 1988.

[9] A. V. Kazhikhov. Recent developments in the global theory of two-dimensional compressible Navier-Stokes equations. Seminar on Mathematical Sciences, Keio University, 25, 1998.

[10] E. J. Koval. A method for predicting the performance of unstable miscible displacements in heterogeneous media. SPEJ trans. AIME, 228:145-154, 1963.

[11] O. A. Ladyzenskaja, V.A. Solonnikov, and N. N. Uralćeva. Linear and Quasi-Linear Equations of Parabolic Type, Translation of Mathematical Monographs, Vol. 23, American Mathematical Society, Providence, RI, 1968.

[12] F. Murat. Compacité par compensation. Ann. Scuola Norm. Sup. Pisa, 5:489-507, 1978.

[13] F. Murat. Soluciones renormalizadas de edp elipticas no lineales. prépublication du Laboratoire d'Analyse Numérique, Université de Paris 6, 93023, 1993.

[14] D. W. Peaceman. Fundamentals of Numerical Reservoir Simulation, Elsevier, 1977.

[15] J. R. A. Pearson and P. M. J. Tardy. Models for flow of non-Newtonian and complex fluids through porous media. J. Non-Newtonian Fluid Mech., 102:447-473, 2002

[16] A. E. Scheideger. The Physics of Flow through Porous Media, Univ. Toronto Press, 1974.

[17] J. Simon. Compact sets in the space $L^{p}(0, T ; B)$. Ann. Math, Pura Appli., IV:65-96, 1987. 
[18] M. R. Todd and W. J. Longstaff. The development, testing and application of a numerical simulator for predicting miscible flood performance. SPEJ trans. AIME, 253:984-998, 1972.

Catherine Choquet

LATP, CNRS UMR 6632

Université P. Cézanne (Aix-Marseille III), FST

Case Cour A

F-13397 Marseille Cedex 20

France

e-mail: c.choquet@univ-cezanne.fr

Received: 21 February 2008.

Accepted: 15 September 2008. 